\title{
MICROLOCAL ANALYSIS OF SOME ISOSPECTRAL DEFORMATIONS
}

\author{
F. MARHUENDA
}

\begin{abstract}
We study the microlocal structure of the examples of isospectral deformations of Riemannian manifolds given by D. DeTurck and C. Gordon in [DeT-G1]. The Schwartz kernel of the intertwining operators considered by them may be written as an oscillatory integral with a singular phase function and product type amplitude. In certain instances, we identify them as belonging to the space of Fourier integral operators associated with various pairwise intersecting Lagrangians. After formulating a class of operators incorporating the most relevant features of the operators above, we establish a composition calculus for this class and show that is not necessary to introduce new Lagrangians in the composition.
\end{abstract}

\section{INTRODUCTION}

Consider a Riemannian manifold $(M, g)$ and its Laplace operator $\Delta$. The question of how much of the geometry of $(M, g)$ is determined by the spectrum of $\Delta$ is a problem in which considerable progress has been made during the last decade. Many new types of nonisometric isospectral Riemannian manifolds have been found. For the history of this interesting subject see [Be, DeT-G1, DeT-G2, D-G, G-W] and the references there.

In $1983 \mathrm{C}$. S. Gordon and E. Wilson exhibited for the first time continuous families of nonisometric, isospectral metrics (isospectral deformations). These occurred on compact solvmanifolds [G-W]. Their examples were further analyzed by D. DeTurck and C. Gordon [DeT-G1] for compact quotients of nilpotent Lie groups of step 2 . In the latter case, operators which intertwine the Laplacians were explicitly found by the authors.

In this work we consider the microlocal structure of the DeTurck-Gordon examples and show that the operators intertwining the Laplacians have a Schwartz kernel that can be put in the form of an oscillatory integral,

$$
\int e^{i \phi(x, y, \theta)} a(x, y, \theta) d \theta,
$$

where the phase function $\phi(x, y, \theta)$ is $C^{\infty}$ in $x$ and $y$ and homogeneous of degree 1 in $\theta$. In this latter variable, it has, at most, singularities of the type $1 / p(\theta)$, where $p(\theta)$ is a fixed homogeneous polynomial in $\theta$. The function $a(x, y, \theta)$ appearing in (1.1) is a product type amplitude. Thus, the kind of

Received by the editors July 3, 1991 and, in revised form, July 31, 1992.

1991 Mathematics Subject Classification. Primary 58G15, 58G25; Secondary 58F05. 
amplitudes needed in the construction of the above operators are more general than Hörmander's symbols.

Away from where the singularity in the phase takes place, the kernel in (1.1) is a Lagrangian distribution associated with two cleanly intersecting Lagrangians. One of these, say $C \subset T^{*} X \backslash 0 \times T^{*} X \backslash 0$, is locally the graph of a canonical map $\chi: T^{*} X \backslash L \rightarrow T^{*} X \backslash L$ which fails to be defined at a certain submanifold $L \subset C$.

We show that in some of the examples, $C$ fails to be a canonical graph at $L$ in a very specific way, namely, along $L$ the projections into the first and second factors, $\pi: T^{*}(X \times X) \rightarrow T^{*} X$ and $\rho: T^{*}(X \times X) \rightarrow T^{*} X$ are blowing-down mappings. Further, $\pi(L)$ and $\rho(L)$ are involutive. Also the contribution from the singularities of the phase in (1.1) introduces a third Lagrangian which is exactly the flow-out from $\pi(L)$.

Motivated by this, we formulate a class of Fourier integral operators associated with canonical relations satisfying the above properties and give a composition calculus and some $L^{2}$ estimates for this class. These operators are not covered by Hörmander's transverse intersection calculus or the DuistermaatGuillemin-Weinstein clean intersection calculus and in fact give rise to distributions in the $I^{p, l}$ classes not considered by Hörmander.

The reason for doing this is to establish the basis for a more ambitious goal: understand completely the above examples from the microlocal point of view and develop a composition calculus that would allow one to construct new examples without making use of the group structure underlying the known cases of isospectral deformations.

One difficulty is that our class of canonical relations cannot be conjugated to a single normal form. In $\S 4$ we show, though, that any such canonical relation is parametrized by a phase function satisfying certain conditions. Then, making use of iterated regularity techniques, we prove the following:

Theorem 1.2. Let $C \subset T^{*}(X \backslash 0) \times T^{*}(Y \backslash 0)$ be a homogeneous canonical relation satisfying: away from a hypersurface $L \subset C$, both projections $\pi: C \rightarrow T^{*}(X)$ and $\rho: C \rightarrow T^{*}(Y)$ are diffeomorphisms, whereas at $L$ they are both blow-downs dropping rank by $k$ and both $\pi(L)$ and $\rho(L)$ are nonradial and involutive. Let $A \in I^{m}(C), B \in I^{m^{\prime}}\left(C^{t}\right)$ be properly supported Fourier integral operators. Then $B A \in I^{m+m^{\prime}+(k-1) / 2,-(k-1) / 2}\left(\Delta_{T^{*} X}, \Lambda_{\pi(L)}\right)$.

Here, $\Delta_{T^{*} X}$ denotes the diagonal in $T^{*} X \times T^{*} X$ and $\Lambda_{\pi(L)}$ the flow-out from $\pi(L)$. As a consequence we also have

Corollary 1.3. For $A$ as above, $A: H^{s}(X) \rightarrow H_{\mathrm{loc}}^{s-m-k / 2}(X)$ is bounded.

It follows from [Hö3] that if the mappings $d \rho$ and $d \pi$ drop rank by at most $k<n$, there is an estimate with a loss of $k / 2$ derivatives: $A: H^{s}(X) \rightarrow$ $H_{\text {loc }}^{s-m-k / 2}(X)$. Thus Corollary 1.3 gives the same estimate, even though $C$ is less singular than a codimension $k$ flow-out. There are examples which show that this estimate is sharp.

Using the same method we also get a composition calculus for $A \in$ $I^{p, l}\left(C, \Lambda_{\pi(L)}\right)$ and $B \in I^{r, s}\left(C^{t}, \Lambda_{\pi(L)}^{t}\right)$. In this case, no new Lagrangian manifolds are introduced.

In all the above results we make extensive use of the iterated regularity char- 
acterization of R. Melrose [Me1, Me2], as developed by Greenleaf and Uhlmann [G-U2, G-U3, G-U4]. A reference in which microlocal analysis has been applied to isospectral problems is [Z].

The author wishes to thank his thesis advisor Allan Greenleaf, to whom he is very deeply indebted for his insights and guidance.

\section{ISOSPECTRAL DEFORMATIONS AND MICROLOCAL ANALYSIS}

In this section we review briefly the examples of [DeT-G1]. The interested reader is referred to the original paper for details.

Let $\mathscr{G}$ be an $n$-dimensional two-step nilpotent Lie algebra, $G=\exp \mathscr{G}$ the associated connected, simply connected Lie group. Then $G$ is diffeomorphic to $R^{n}$ for some $n$ and $[\mathscr{G}, \mathscr{G}] \subset \mathscr{Z}$, the center of $\mathscr{G}$. We denote the adjoint representation of $\mathscr{G}$ by $\operatorname{ad}_{Y}(X)=[Y, X]$. A map $\phi: \mathscr{G} \rightarrow \mathscr{Z}$ is called an almost-inner derivation [G-W] if for each $X \in \mathscr{G}$ there is $Y \in \mathscr{G}$, possibly depending on $X$, such that $\phi(X)=\operatorname{ad}_{Y}(X)$.

Two consequences of this definition are that $\phi(\mathscr{Z})=0$ and that $\phi$ is a derivation (since $\mathscr{G}$ is two-step nilpotent). For nilpotent Lie groups with higher step one needs to impose also that $\phi$ is a derivation. This derivation is inner if and only if one can choose a $Y \in \mathscr{G}$ which does not depend on $X$ in the above definition (thus if $\phi=\operatorname{ad}_{Y}$ ).

We can exponentiate $\phi$ to get an automorphism of $\mathscr{G}$. By the previous remark, $\phi^{2}=0$ so $\Phi=\exp \phi=I+\phi$ and the map from $\operatorname{Aut}(G)$ to $\operatorname{Aut}(\mathscr{G})$ that assigns to an automorphism $\Phi: G \rightarrow G$ its derivative at the identity $\Phi_{*}$, is an isomorphism. Thus, to $\exp \phi$ there corresponds an automorphism of $G$ for which we use the same name. Since $G$ is two-step nilpotent, the BakerCampbell-Hausdorff formula provides us with a convenient description of this map: if $X=\log (x)$ then $\Phi(x)=\exp (X+\phi(X))$. Note that the derivative of the latter at the identity is $\Phi=I+\phi$ and it satisfies

$$
\begin{aligned}
\Phi(x y) & =\exp \Phi(\log (x y))=\exp \left(\Phi\left(X+Y+\frac{1}{2}[X, Y]\right)\right) \\
& =\exp \left(X+Y+\frac{1}{2}[X, Y]+\phi(X)+\phi(Y)\right) \\
& =\exp (X+\phi(X)) \exp (Y+\phi(Y)) .
\end{aligned}
$$

Lemma 2.1 [DeT-G1]. Let $\phi$ be a derivation of a two-step nilpotent Lie algebra $\mathscr{G}$, and let $\mathscr{Z}$ be the center of $\mathscr{G}$. Then $\phi$ is almost-inner if and only if, for every linear functional $\lambda \in \mathscr{Z}^{*}$, there exists a vector $Y_{\lambda} \in \mathscr{G}$ such that $\lambda \circ \phi=\lambda \circ \operatorname{ad}_{Y_{\lambda}}$, i.e.,

$$
\lambda(\phi(X))=\lambda\left(\left[Y_{\lambda}, X\right]\right)
$$

for all $X \in \mathscr{G}$.

A subalgebra $\mathscr{L}$ of $\mathscr{G}$ is called $\phi$-admissible if it contains $\mathscr{Z}$ (and therefore $\mathscr{L}$ is an ideal of $\mathscr{G}$ ) and some choice of $Y_{\lambda}$ satisfying (2.2) for each $\lambda \in \mathscr{Z}^{*}$. Likewise, $\exp \mathscr{L}$ will be called a $\phi$-admissible subgroup of $G$.

Let $\phi$ be an almost-inner derivation of $\mathscr{G}$ that is not inner and choose a $\phi$-admissible subalgebra $\mathscr{L}_{\phi}$ of $\mathscr{G}$ which is the Lie algebra of the subgroup $H_{\phi}=\exp \mathscr{L}_{\phi}$. Let $\Gamma$ be a uniform discrete subgroup of $G$, so that $\Gamma \backslash G$ is compact. Let $\mathscr{M}$ be the set of metrics on $G$ that are left-invariant under the action of the subgroup $\Gamma H_{\phi}$. The Riemannian metric $g \in \mathscr{M}$ induces 
a unique Riemannian metric (also called $\mathrm{g}$ ) on $\Gamma \backslash G$ such that the projection $\pi:(G, g) \rightarrow(\Gamma \backslash G, g)$ is a Riemannian covering.

Theorem 2.3 [DeT-G1]. Let $G, \mathscr{G}, \Gamma, g, \phi$, and $\mathscr{L}_{\phi}$ be as above, and define $\Phi_{t} \in \operatorname{Aut}(G)$ by $\Phi_{t}=\exp (t \phi)=I+t \phi$. Then, for every $t, g_{t}=\Phi_{t}^{*} g$ is also left invariant by $\Gamma H_{\phi}$ and the family $\left\{g_{t}\right\}_{t \in R}$ is a continuous isospectral deformation of $g$ on $\Gamma \backslash G$. The deformation is nontrivial for all $g$ in some nonempty open subset of $\mathscr{M}$.

In the proof of Theorem 2.3, the authors in [DeT-G1] construct an operator, $S_{t}$ that intertwines the Laplacians, i.e., $S_{t}$ satisfies

$$
S_{t}^{-1} \Delta S_{t}=\Delta_{t}, \quad t \geq 0,
$$

where $\Delta_{t}$ is the Laplacian associated with $g_{t}$. In this work, we study these operators from the microlocal point of view. We proceed next to describe them more precisely.

Let $Z=\exp \mathscr{Z}$ be the center of the group. The map exp induces an isomorphism of abelian groups from $(\mathscr{Z} \cap \log (\Gamma)) \backslash \mathscr{Z}$ onto the torus $T=(Z \cap \Gamma) \backslash Z$. The center, $Z$, and the torus $T$ act on $\mathscr{H}=L^{2}(\Gamma \backslash G)$ by left translation: for $f \in \mathscr{H}, z \in Z$, the action of $Z$ sends $f$ to $L_{z}^{*}(f)$. Hence it is possible to extend the ordinary Fourier series decomposition of $L^{2}(T)$ to one of $L^{2}(\Gamma \backslash G)$ by writing $\mathscr{H}=\bigoplus_{\left\{\lambda \in \mathscr{Z}^{*}: \lambda(\mathscr{E} \cap \log (\Gamma) \in \mathscr{Z}\}\right.} \mathscr{H}_{\lambda}$, where

$$
\mathscr{H}_{\lambda}=\left\{f \in \mathscr{H}: L_{z}^{*} f=e^{2 \pi i \lambda(\log z)} f \quad \forall z \in Z\right\} .
$$

Note that $\Delta: \mathscr{H}_{\lambda} \rightarrow \mathscr{H}_{\lambda}$ since, for $z \in Z$, the Laplacian $\Delta$ commutes with the isometries $L_{z}$. Define $S_{\lambda, t}=\left(L_{y_{\lambda, t}} \circ \Phi_{-t}\right)^{*}$ on $\mathscr{L}_{\lambda}$, where $y_{\lambda, t}=\exp \left\{t Y_{\lambda}\right\}$ and $S_{t}=\bigoplus_{\lambda} S_{\lambda, t}$. The operator $S_{t}$ is continuous on $L^{2}(\Gamma \backslash G)$ with respect to the topology induced by the norm corresponding to a left $G$-invariant metric on $\Gamma \backslash G$. It is shown in [DeT-G1] that $\left(L_{y_{\lambda, t}} \circ \Phi_{-t}\right)^{*}=R_{y_{\lambda, t}}^{*}$ on each of the spaces $\mathscr{H}_{\lambda}$.

It is also shown in [DeT-G1] that this operator does indeed satisfy (2.4) and that this deformation is nontrivial whenever $\phi$ is not inner and the metric $g$ is close enough to the space of metrics invariant under the action of the group.

The map $\lambda \mapsto Y_{\lambda}$ may not be unique, but (2.2) imposes rather strict conditions on it. Let $\left\{Z_{1}, \ldots, Z_{m}\right\}$ be a basis for the center of $\mathscr{Z}$ and complete it with $\left\{X_{1}, \ldots, X_{n}\right\}$ to form a basis for $\mathscr{G}$. With this choice, $\mathscr{G}$ splits into $\mathscr{G}=\mathscr{N} \oplus \mathscr{Z}$. This induces a (smooth) product structure on $G$. We will make use of $x=\left(x_{1}, \ldots, x_{n}\right)$, and $z=\left(z_{1}, \ldots, z_{m}\right)$ to denote the coordinates corresponding to $\exp (N)$ and $\exp (Z)$, respectively. In $\mathscr{Z}^{*}$ we use coordinates $\left(\lambda_{1}, \ldots, \lambda_{n}\right)$ dual to $\left\{Z_{1}, \ldots, Z_{m}\right\}$.

Adding to $Y_{\lambda}$ an element of the center will not affect (2.2) so we may assume

$$
Y_{\lambda}=\sum_{i=1}^{n} a_{i}(\lambda) X_{i}
$$

Let $\left\{C_{i j}^{k}\right\}_{i, j . k}$ be the structure constants, i.e.,

$$
\left[X_{i}, X_{j}\right]=\sum_{k=1}^{m} C_{i j}^{k} Z_{k}
$$


Then $\left[Y_{\lambda}, X_{j}\right]=\sum_{i, k} a_{i} C_{i j}^{k} Z_{k}$ and in this basis the matrix representing $\operatorname{Ad}_{Y_{\lambda}}$ is

$$
\operatorname{Ad} Y_{\lambda}=\left(\begin{array}{cccc}
\sum_{i} a_{i} C_{i 1}^{1} & \sum_{i} a_{i} C_{i 2}^{1} & \ldots & \sum_{i} a_{i} C_{i n}^{1} \\
\vdots & \vdots & \ddots & \vdots \\
\sum_{i} a_{i} C_{i 1}^{m} & \sum_{i} a_{i} C_{i 2}^{m} & \ldots & \sum_{i} a_{i} C_{i n}^{m}
\end{array}\right)
$$

By (2.2), the map $\lambda \mapsto \lambda \circ \operatorname{Ad}_{Y_{\lambda}}$ is linear, hence the following expressions are linear in $\lambda$ :

$$
\begin{gathered}
\lambda_{1} \sum_{i} a_{i} C_{i 1}^{1}+\lambda_{2} \sum_{i} a_{i} C_{i 1}^{2}+\cdots+\lambda_{m} \sum_{i} a_{i} C_{i 1}^{m}, \\
\lambda_{1} \sum_{i} a_{i} C_{i 2}^{1}+\lambda_{2} \sum_{i} a_{i} C_{i 2}^{2}+\cdots+\lambda_{m} \sum_{i} a_{i} C_{i 2}^{m}, \\
\vdots \\
\lambda_{1} \sum_{i} a_{i} C_{i n}^{1}+\lambda_{2} \sum_{i} a_{i} C_{i n}^{2}+\cdots+\lambda_{m} \sum_{i} a_{i} C_{i n}^{m}
\end{gathered}
$$

As a consequence the functions $a_{i}(\lambda)$ must have the form

$$
a_{i}(\lambda)=q_{i}(\lambda) / p(\lambda),
$$

where $q_{i}(\lambda)$ and $p(\lambda)$ are homogeneous polynomials in $\lambda$ of the same degree.

Next, we look at the construction of the operator $S_{t}$ from the microlocal point of view. $G$ has a local product structure coming from the splitting of its Lie algebra $\mathscr{G}=\mathscr{N} \oplus \mathscr{Z}$ mentioned above. Fix a point $p \in \Gamma \backslash G$. Let $\pi: G \rightarrow \Gamma \backslash G$ denote the projection onto $\Gamma \backslash G$. The map exp : $\mathscr{G} \rightarrow G$ is a global diffeormorphism. Thus, for appropriately chosen neighborhoods $\pi \circ \exp$ provides a local chart around $p$. Locally, in the given coordinate chart, the manifold $M=\Gamma \backslash G$ is also a product of manifolds $N \times Z$.

In the above basis, we can identify $G$ and $\mathscr{G}$ with $\mathbb{R}^{n} \times \mathbb{R}^{m}$ and express the product in the group $G$ as follows: Recall that we use $(x, z) \in \mathbb{R}^{n} \times \mathbb{R}^{m}=G$ to denote points in the group. There is a bilinear form $B: \mathbb{R}^{n} \times \mathbb{R}^{n} \rightarrow \mathbb{R}^{m}$, such that for $(x, z),(y, c) \in G$, their product is given by $L_{(x, z)}(y, c)=(x, z) \cdot(y, c)=$ $(x+y, z+c+B(x, y))$. By making use of the above basis we may write the bilinear form $B$ as $B(x, y)=\left(\sum_{i, j=1}^{n} B_{i j}^{1} x_{i} y_{j}, \ldots, \sum_{i, j=1}^{n} B_{i j}^{m} x_{i} y_{j}\right)$.

With the above notation, the map $S_{\lambda, t}$ maps the function $f(x, z) \in \mathscr{L}_{\lambda}$ to $f\left(x+t y_{\lambda}, z+t B\left(y_{\lambda}, x\right)-t \phi(x)\right)=f\left(x+t y_{\lambda}, z+t B\left(x, y_{\lambda}\right)\right)$, since $L_{y_{\lambda}} \circ \phi_{t}=R_{y_{\lambda}}$ on $\mathscr{H}_{\lambda}$. form

We remark here that a function $f(x, z)$ is in $\mathscr{H}_{\lambda}$ if and only if it has the

$$
f(x, z)=g(x) e^{2 \pi i \lambda \cdot z},
$$

with $g$ satisfying that for each $\sigma \in \Gamma, g(x)=g(x+\sigma) e^{2 \pi i \lambda \cdot B(\sigma, x)}$.

Define now the function

$$
F(\zeta, x, t)=x+t y_{\zeta}
$$

and consider the following phase:

$$
\psi(x, y, \xi ; z, w, \zeta)=(F(\zeta, x, t)-y) \cdot \xi+\left(z-w+t B\left(x, y_{\zeta}\right)\right) \cdot \zeta
$$


For $H \in L^{1}(G)$, we define the operator

$$
\left(T_{t} H\right)(x, z)=\int e^{i \psi(x, y, \xi ; z, w, \zeta)} H(y, w) d \xi d \zeta d y d w
$$

We have

Proposition 2.12. The operators $T_{t}$, defined by equation (2.11), and $S_{t}$ have the same microlocal form.

Proof. In the following, we will use the notation $\mathscr{F}(f)(x, a), \mathscr{F}(H)(x, \zeta)$ (where $a \in \mathbb{Z}^{n}, \zeta \in \mathbb{R}^{n}$ ) to denote Fourier transformation in the second variable (in the appropriate spaces).

Note, firstly, that for any function $H$, compactly supported in $G$, we have

$$
\begin{aligned}
& \mathscr{F}\left(T_{t} H\right)(x, \gamma)=\int e^{i(\psi(x, y, \xi ; z, w, \zeta)-\gamma \cdot z)} H(y, w) d \xi d \zeta d z d y d w \\
& =\int e^{i\left((F(\zeta, x, t)-y) \cdot \xi+\left(t B\left(x, y_{\zeta}\right)-w\right) \cdot \zeta\right)}\left(\int e^{i(z-\gamma) \cdot \zeta} d z\right) H(y, w) d \xi d \zeta d y d w .
\end{aligned}
$$

Since $\int e^{i(z-\gamma) \cdot \zeta} d z=\delta_{\gamma}(\zeta)$, Dirac's delta function, we obtain

$$
\begin{aligned}
\mathscr{F}\left(T_{t} H\right)(x, \gamma) & =\int e^{i\left((F(\gamma, x, t)-y) \cdot \xi+\left(t B\left(x, y_{\gamma}\right)-w\right) \cdot \gamma\right)} H(y, w) d \xi d y d w \\
& =e^{i t B\left(x, y_{\gamma}\right) \cdot \gamma} \int H(F(\gamma, x, t), w) e^{-i w \cdot \gamma} d w \\
& =e^{i t B\left(x, y_{\gamma}\right) \cdot \gamma \mathscr{F}(H)(F(\gamma, x, t), \gamma) .}
\end{aligned}
$$

Let now $h \in L^{1}(\Gamma \backslash G)$. Identifying functions on the quotient space with periodic functions on the cover, we may assume $h$ is defined on $G$, and satisfies

$$
h((\gamma, c) \cdot(x, z))=h((x+\gamma, z+c+B(\gamma, x)))=h(x, z),
$$

for all $(\gamma, c) \in \Gamma$ (i.e., $h$ is periodic). In particular,

$$
h(x, z+c)=h(x, z),
$$

if $(0, c) \in \Gamma \cap Z$. And we may apply the usual Fourier analysis to it. We remark that, in our notation, the map exp: $\mathscr{G} \rightarrow G$ is given by $\exp (x, z)=$ $\left(x, z+\frac{1}{2} B(x, x)\right)$, and hence, it induces the identity map $\left.\exp \right|_{\mathscr{Z}}: \mathscr{Z} \rightarrow Z$. Identifying these two spaces we may also identify $\mathscr{Z}^{*}=\mathbb{R}^{m *}$.

There is, now, another function $H(x, z)$, defined on $G$, with compact support in the $z$ variable, and such that, with the above identification,

$$
h(x, z)=\sum_{a \in Z \cap \Gamma} H\left(L_{a}(x, z)\right) .
$$

Note that if $a \in Z \cap \Gamma$ then $a$ must be of the form $a=(0, c)$. And, hence, $L_{a}(x, z)=(x, z+c)$. Making a Fourier decomposition in the second variable, we obtain

$$
h(x, z)=\sum_{\gamma \in \mathbf{R}^{m *} \cap \Gamma^{*}} a_{\gamma}(x) e^{i z \cdot \gamma}
$$


where $\Gamma^{*}=\left\{\gamma \in \mathbb{R}^{m *}: \forall a \in \Gamma \cap Z, \gamma(a) \in \mathbb{Z}\right\}$ and $a_{\gamma}(x)=\int H(x, y) e^{-i y^{*} \gamma} d y$. (Here, we are identifying $y \cdot \gamma=\gamma(y)$.) Thus the operator $S_{t}$ can be written as

$$
\begin{aligned}
\left(S_{t} h\right)(x, z) & =\sum_{\gamma \in \mathbb{R}^{m *} \cap \Gamma^{*}} a_{\gamma}(F(\gamma, x, t)) e^{i\left(z+t B\left(x, y_{\gamma}\right)\right) \cdot \gamma} \\
& =\sum_{\gamma \in \mathbb{R}^{m *} \cap \Gamma^{*}} a_{\gamma}(F(\gamma, x, t)) e^{i t B\left(x, y_{\gamma}\right) \cdot \gamma} e^{i z \cdot \gamma} .
\end{aligned}
$$

This shows that the Fourier coefficients of $S_{t}(h)$ are

$$
\mathscr{F}\left(S_{t} h\right)(x, \gamma)=a_{\gamma}(F(\gamma, x, t)) e^{i t B\left(x, y_{\gamma}\right) \cdot \gamma}=\mathscr{F}(h)(F(\gamma, x, t), \gamma) e^{i t B\left(x, y_{\gamma}\right) \cdot \gamma} .
$$

But from the Poisson formula we have that

$$
\mathscr{F}(h)(F(\gamma, x, t), \gamma)=\mathscr{F}(H)(F(\gamma, x, t), \gamma),
$$

and, hence,

$$
\mathscr{F}\left(S_{t}(h)\right)(F(\gamma, x, t), \gamma)=\mathscr{F}\left(T_{t}(H)\right)(F(\gamma, x, t), \gamma) .
$$

Since, for fixed $\gamma \in \mathbb{R}^{m *} \cap \Gamma^{*}$, the mapping $F(\gamma, x, t)$ is a diffeomorphism, we see that $T_{t}(H)$ and $S_{t}(h)$ have the same Fourier coefficients and the proposition follows.

For a general $C^{\infty}$ function in $\Gamma \backslash G$ with support not necessarily contained in the coordinate chart above, we use a microlocal partition of unity, $C^{\infty}$ in the spatial variables, homogeneous in the cotangent variables, involving first the $(z, \zeta)$ and then the $(x, \xi)$ directions. Thus the resulting amplitude will have a product type nature as in [M-U, Gu-U].

Making use of this partition of unity to first localize the functions, and then applying the operator $T_{t}$ in (2.11) will not change its microlocal structure. Thus, the resulting operators $T_{t}$, will have Schwartz kernels with the following microlocal form:

$$
K(x, z, y, w)=\int e^{i\left[\xi \cdot(F(\zeta, x, t)-y)+\zeta \cdot\left(z-w+t B\left(x, y_{\zeta}\right)\right)\right]} a(x, y ; \xi, \zeta) d \xi d \zeta
$$

where $a(x, y ; \xi, \zeta)$ is a product type amplitude.

One can interpret the examples in [DeT-G1] from this point of view.

Example 2.15 (from [DeT-G1]). Consider a Lie algebra with $n=4, m=2$ and the following structure constants $C_{13}^{1}=C_{24}^{1}=C_{14}^{2}=1$ and all the other $C_{j k}^{i}$ vanish. For the almost inner derivation $\phi$ which maps $X_{1}$ to $Z_{2}$ and vanishes on all the other elements of the basis, one obtains

$$
\begin{aligned}
& a_{1}(\lambda)=a_{2}(\lambda)=0, \\
& a_{3}(\lambda)= \begin{cases}-\lambda_{2} / \lambda_{1} & \text { if } \lambda_{1} \neq 0, \\
0 & \text { if } \lambda_{1}=0,\end{cases} \\
& a_{4}(\lambda)= \begin{cases}0 & \text { if } \lambda_{1} \neq 0, \\
-1 & \text { if } \lambda_{1}=0 .\end{cases}
\end{aligned}
$$

Thus,

$$
\begin{aligned}
& F(\zeta, x, t)=\left(x_{1}, x_{2}, x_{3}-t \frac{\zeta_{2}}{\zeta_{1}}, x_{4}\right) \\
& H(\zeta, x, t)=\left(-t x_{1} \frac{\zeta_{2}}{\zeta_{1}}, 0\right), \quad \zeta \cdot H(\zeta, x, t)=-t x_{1} \zeta_{2}=\zeta \cdot\left(0,-t x_{1}\right),
\end{aligned}
$$


and we obtain

$$
K(x, z ; y, w)=\int e^{i \psi(x, z ; y, w ; \xi, \zeta)} d \xi d \zeta
$$

with

$$
\psi(x, z ; y, w ; \xi, \zeta)=(x-y) \xi-t \frac{\zeta_{2}}{\zeta_{1}} \xi_{3}+\left(z_{1}-w_{1}\right) \zeta_{1}+\left(z_{2}-w_{2}-t x_{1}\right) \zeta_{2} .
$$

\section{PSEUDODIFFERENTIAL OPERATORS WITH SINGULAR SYMBOLS}

The microlocal structure of operators of the form (2.14) is related to the spaces of distributions associated with cleanly intersecting Lagrangian manifolds introduced in [M-U] and [Gu-U]. These spaces have been further studied in [A$\mathrm{U}, \mathrm{G}-\mathrm{U} 1, \mathrm{G}-\mathrm{U} 2, \mathrm{G}-\mathrm{U} 3, \mathrm{G}-\mathrm{U} 4, \mathrm{Gu} 1]$. In this section we review some of the results pertaining to our case.

Let $X$ and $Y$ denote $C^{\infty}$ manifolds of dimension $n$. Denote by $\omega_{X}=$ $\sum_{i=1}^{n_{X}} d \xi_{i} \wedge d x_{i}$ (resp. $\omega_{Y}$ ) the canonical symplectic two-form on $T^{*} X$ (resp. $\left.T^{*} Y\right)$. On $T^{*}(X \times Y)$ we take the canonical symplectic two-form $\omega_{X}-\omega_{Y}$. Let $\pi: T^{*}(X \times Y) \rightarrow T^{*} X$ and $\rho: T^{*} X \times T^{*} Y \rightarrow T^{*} Y$ be the canonical projections.

Recall that two Lagrangian submanifolds, say $L$ and $K$, of a symplectic manifold $M$, intersect cleanly [Bo] if $L \cap K$ is a manifold and for each $p \in$ $L \cap K$ we have $T_{p}(L \cap K)=T_{p} L \cap T_{p} K$. Since only the case $\Lambda_{0}, \Lambda_{1} \subset T^{*}(X \times Y)$ is relevant for our purposes, we will adapt the results to this particular situation.

We start by considering the model case in which $X=Y=\mathbb{R}^{n}$, with coordinates $(x, \xi), \tilde{\Lambda}_{0}=\Delta_{T^{*}\left(\mathbb{R}^{n} \times \mathbb{R}^{n}\right)}$ is the diagonal in $T^{*}(X \times Y)$ and

$$
\tilde{\Lambda}_{1}=\left\{(x, \xi ; y, \eta): x^{\prime \prime}=y^{\prime \prime}, \xi^{\prime}=\eta^{\prime}=0, \xi^{\prime \prime}=\eta^{\prime \prime}\right\}
$$

is the flow-out from $\tilde{\Lambda}_{0} \cap\left\{\xi^{\prime}=0\right\}$. We are using the notation $x=\left(x^{\prime}, x^{\prime \prime}\right) \in$ $\mathbb{R}^{k} \times \mathbb{R}^{n-k}$. These two Lagrangians intersect cleanly at $\Sigma=\{x=y, \xi=$ $\left.\eta, \xi^{\prime}=0\right\}$. We define the class $I^{p, l}\left(\tilde{\Lambda}_{0}, \tilde{\Lambda}_{1}\right)$ to be the space of operators whose Schwartz kernels have the form

$$
K_{A}(x, y)=\int e^{i\left[\left(x^{\prime}-y^{\prime}-s\right) \cdot \theta^{\prime}+\left(x^{\prime \prime}-y^{\prime \prime}\right) \cdot \theta^{\prime \prime}+s^{*} \sigma\right]} a(x, y, s ; \theta, \sigma) d \theta d \sigma d s,
$$

where $a(x, y, s ; \theta, \sigma)$ is a product type symbol of order $p^{\prime}=p-n / 2+$ $k / 2$ in $\theta$ and $l^{\prime}=l-k / 2$ in $\sigma$, i.e., it satisfies that for each compact set $K \subset \mathbb{R}^{n} \times \mathbb{R}^{n} \times \mathbb{R}^{k}$ and multi-indices $\alpha, \beta, \gamma, \delta, \epsilon$, there is a constant $C=C(K, \alpha, \beta, \gamma, \delta, \epsilon)$ such that for $(x, y, s) \in K$,

$$
\left|\partial_{\theta}^{\alpha} \partial_{\sigma}^{\beta} \partial_{s}^{\gamma} \partial_{x}^{\delta} \partial_{y}^{\epsilon} a(x, y, s ; \theta, \sigma)\right| \leq C(1+|\theta|)^{p^{\prime}-|\alpha|}(1+|\sigma|)^{l^{\prime}-|\beta|} .
$$

We have [Gu-U].

Proposition 3.3. Let $\tilde{\Lambda}_{0}, \tilde{\Lambda}_{1}$, and $\Sigma$ be as above. Then $W F\left(K_{A}\right) \subset \tilde{\Lambda}_{0}^{\prime} \cup \tilde{\Lambda}_{1}^{\prime}$. Moreover, microlocally near $\tilde{\Lambda}_{0} \backslash \Sigma, K_{A}$ is in the space $I^{p+l}\left(\tilde{\Lambda}_{0} \backslash \Sigma\right)$. Near $\tilde{\Lambda}_{1} \backslash \Sigma$ it is microlocally in the space $I^{p}\left(\tilde{\Lambda}_{1} \backslash \Sigma\right)$.

For general $X, Y$, and conic Lagrangian submanifolds $\Lambda_{0}$ and $\Lambda_{1} \subset$ $T^{*} X \backslash 0 \times T^{*} Y \backslash 0$ intersecting cleanly in a codimension $k$ submanifold, there exists [Gu-U] a locally finite covering of $\Lambda_{0} \cap \Lambda_{1}$ by conic sets $\left\{U_{i}\right\}$ such 
that for each $U_{i}$ there is a homogeneous canonical transformation $\chi_{i}: U_{i} \rightarrow$ $T^{*} \mathbb{R}^{n} \backslash 0 \times T^{*} \mathbb{R}^{n} \backslash 0$ mapping $U_{i} \cap \Lambda_{0}$ into $\tilde{\Lambda}_{0}$ and $U_{i} \cap \Lambda_{1}$ into $\tilde{\Lambda}_{1}$.

The space $I^{p, l}\left(\Lambda_{0}, \Lambda_{1}\right)$ consists of all distributions $\mu$ on $X \times Y$ which can be written in the form

$$
\mu=\mu_{0}+\mu_{1}+\sum \omega_{i}
$$

where $\mu_{0} \in I^{p+l}\left(\Lambda_{0} \backslash \Sigma\right), \mu_{1} \in I^{p}\left(\Lambda_{1} \backslash \Sigma\right)$ and $\omega_{i}$ is microlocally supported in $U_{i}$ and is of the form $F_{i} \nu_{i}$, where $\nu_{i} \in I^{p, l}\left(\tilde{\Lambda}_{0}, \tilde{\Lambda}_{1}\right)$ and $F_{i}$ is a zeroth-order operator associated with $\chi^{-1}$.

One can show that if $\chi:\left(T^{*} \mathbb{R}^{n} \backslash 0 \times T^{*} \mathbb{R}^{n} \backslash 0\right) \rightarrow\left(T^{*} \mathbb{R}^{n} \backslash 0 \times T^{*} \mathbb{R}^{n} \backslash 0\right)$ is a homogeneous canonical transformation which leaves both $\tilde{\Lambda}_{0}$ and $\tilde{\Lambda}_{1}$ invariant, $\mu$ is in $I^{p, l}\left(\Lambda_{0}, \Lambda_{1}\right)$ and $F$ is a zeroth-order Fourier integral operator associated with $\chi$, then $F \mu$ is in $I^{p, l}\left(\Lambda_{0}, \Lambda_{1}\right)$.

The oscillatory representation (3.1) is usually difficult to verify in practice. We will be making use of two other characterizations of these spaces. The first one is a variation, given by A. Greenleaf and G. Uhlmann, of the iterated regularity characterizations of $\mathrm{R}$. Melrose.

Proposition 3.4 ([G-U2]; see also [Me1] and [Me2]). Let $\Lambda \subset\left(T^{*} X \backslash 0\right) \times$ $\left(T^{*} X \backslash 0\right)$ be a canonical relation cleanly intersecting the diagonal $\Delta$. Then $u \in$ $I^{p, l}(\Delta, \Lambda)$ for some $p, l \in \mathbb{R}$ if and only if for some $s_{0} \in \mathbb{R}$, all $k \geq 0$, and all first-order pseudodifferential operators $P_{1}\left(z, D_{z}, y, D_{y}\right), \ldots, P_{k}\left(z, D_{z}, y, D_{y}\right)$, characteristic for $\Delta^{\prime} \cup \Lambda^{\prime}$,

$$
P_{1} \cdots P_{k} u \in H_{\mathrm{loc}}^{s_{0}}(X \times X) .
$$

A multiphase [Mn] parametrizing the pair $\Lambda_{0}, \Lambda_{1}$ is a phase function $\phi(x, y, s, \theta)$ on $X \times Y \times R^{j} \times R^{N}$, smooth in $x, y$, and $s$ and homogeneous of degree 1 in $\theta$, such that

$$
\phi_{0}(x, y, \theta)=\phi(x, y, 0, \theta)
$$

parametrizes $\Lambda_{0}$ and

$$
\phi_{1}(x, y, \sigma, \theta)=\phi(x, y, \sigma /|\theta|, \theta)
$$

parametrizes $\Lambda_{1}$.

The second characterization, by Greenleaf and Uhlmann [G-U4], is by means of oscillatory integrals with multiphase.

Proposition 3.6. A distribution $u \in I^{p, l}\left(\Lambda_{0}, \Lambda_{1}\right)$ iff it can be written in the form

$$
u(x, y)=\iint_{R^{j} \times R^{N}} e^{i \phi(x, y, \sigma /|\theta|, \theta)} a(x, y, \sigma, \theta) d \sigma d \theta
$$

with $\phi(x, y, s, \theta)$ a multiphase parametrizing $\Lambda_{0}, \Lambda_{1}$, and $a(x, y, \sigma, \theta) a$ product type symbol of order $p+l-n / 2+j / 2$ in $\theta$ and $-l-j+n-N / 2$ in $\sigma$.

We apply now the above calculus to operators of the form (2.14). We let $X=Y=G$. Making use of the multiphase

(3.8) $\Phi(x, z ; y, w ; s ; \theta)=|\theta|(F(\theta, x, t)-y) \cdot s+\left(z-w+t B\left(x, y_{\zeta}\right)\right) \cdot \theta$,

and Proposition 3.6, we see that microlocally away from the region

$$
L=\left\{(x, z, \xi, \zeta ; y, w, \eta, \omega) \in T^{*} G \times T^{*} G: p(\zeta)=0\right\}
$$


operators given by equation (2.11) are Lagrangian distributions associated with the Lagrangian manifolds $\Lambda_{0}$ and $\Lambda_{1}$ of $T^{*} G \times T^{*} G$ parametrized, respectively, by the phases

$\Phi_{0}=\Phi(x, z ; y, w ; 0, \theta)=\theta \cdot\left(z-w+t B\left(x, y_{\zeta}\right)\right)=\theta \cdot\left(z-w+t B\left(x, y_{\zeta}\right)-t \phi(x)\right)$

and

$$
\Phi_{1}=\Phi(x, z ; y, w ; \xi|\zeta|, \zeta)=\xi \cdot(F(\zeta, x, t)-y)+\zeta \cdot\left(z-w+t B\left(x, y_{\zeta}\right)\right) .
$$

In particular, $\Lambda_{1}$ is locally the graph of a canonical transformation

$$
\chi: T^{*} G \backslash L \rightarrow T^{*} G \backslash L .
$$

In the following we will assume that $y_{\lambda}$ has the form

$$
y_{\lambda}=\frac{1}{\lambda_{1}}(\underbrace{0, \ldots, 0}_{n-k}, b_{n-k+1} \lambda_{p_{n-k+1}}, \ldots, b_{n} \lambda_{p_{n}}),
$$

where $b_{j} \in R \backslash\{0\}$. Hence, the phase in (2.10) is then

$$
\begin{aligned}
\psi(x, y, \xi ; z, w, \zeta)= & \sum_{i=1}^{n-k}\left(x_{i}-y_{i}\right) \xi_{i}+\sum_{i=n-k+1}^{n}\left(x_{i}-y_{i}+t b_{i} \frac{\zeta_{p_{i}}}{\zeta_{1}}\right) \xi_{i} \\
& +(z-w) \cdot \zeta+\frac{t}{\zeta_{1}} \sum_{l=1}^{m} \sum_{i=1}^{n} \sum_{j=n-k+1}^{n} B_{i j}^{l} b_{j} x_{i} \zeta_{p_{j}} \zeta_{l}
\end{aligned}
$$

We will further assume that

$$
B\left(y_{\lambda}, x\right)=0 \text {. }
$$

In general, one expects a third contribution to the wavefront set of $K(x, z, y, w)$ in (2.14), coming from the singularities of the phase which occur in the region $p(\theta)=0$. The contributions from this region might be very complicated. However, there are cases when it is possible to describe them. The hypothesis in the proposition below is satisfied by some of the examples in [DeT-G1].

Proposition 3.12. (1) If assumption (3.9) holds, then $\Lambda_{1}$ can be extended past $L$. Further, $\Lambda_{1}$ fails to be a canonical graph at $L$ in a very specific way: the mappings $\pi: \Lambda_{1} \rightarrow T^{*} G$ and $\rho: \Lambda_{1} \rightarrow T^{*} G$ are blow-downs (defined below) along $L$ and both $\pi(L)$ and $\rho(L)$ are involutive.

(2) If, in addition, assumption (3.11) holds, then one obtains a third contribution, $\Lambda_{2}=\Lambda_{\pi(L)}$, to the wavefront set of $K(x, z, y, w)$ in (2.14), which is the flow-out from $\pi(L)$.

Proof. Let us assume first (3.9). From the usual microlocal calculus it follows that, microlocally away from $L, \Lambda_{1}$ is the set

$$
\begin{aligned}
\Lambda_{1}=\left\{(x, z, \xi, \zeta ; y, w, \eta, \omega): d_{\xi} \psi=d_{\zeta} \psi\right. & =0, \xi=d_{x} \psi, \\
\zeta=d_{z} \psi, \eta & \left.=d_{y} \psi, \omega=d_{w} \psi\right\},
\end{aligned}
$$


where $\psi$ is given by (3.10) and $L$ is the set

$$
L=\left\{(x, z, \xi, \zeta ; y, w, \eta, \omega) \in \Lambda_{1}: \zeta_{1}=0\right\}
$$

After a tedious, but standard, computation it follows that $\Lambda_{1}$ is the conormal bundle to the following submanifold of $G \times G$ :

$$
\begin{aligned}
& S_{1}=\left\{(x, z ; y, w) \in G \times G: y_{i}=x_{i}, i=1, \ldots, n-k,\right. \\
& w_{h}=z_{h}-B^{h}(x, x-y), h \notin\left\{1, p_{n-k}, \ldots, p_{n}\right\}, \\
& \left.w_{1}=z_{1}-\frac{1}{t} \sum_{h=n-k+1}^{n} \frac{x_{h}-y_{h}}{b_{h}}\left(z_{p_{h}}-w_{p_{h}}-B^{p_{h}}(x, x-y)\right)-B^{1}(x, x-y)\right\} .
\end{aligned}
$$

This parametrization does not have any singularities as we approach $L$. Writing now local coordinates for $\Lambda_{1}$ and using the above characterization, it follows easily that the mappings $\pi: \Lambda_{1} \rightarrow T^{*} G$ and $\rho: \Lambda_{1} \rightarrow T^{*} G$ are blow-downs, dropping rank by $n-k$ at $L$. It is also immediate to check using the above coordinates that $\pi(L)$ and $\rho(L)$ are defined by an involutive set of defining functions.

If we further assume (3.11), then we may rewrite $\Phi_{0}$ as $\Phi_{0}=\theta \cdot(z-w-$ $t \phi(x))$, which is a linear phase. Hence, $\Lambda_{0}$ is the conormal bundle to the graph of $\phi_{t}(x)$. Writing local coordinates and using the above set of defining functions for $S_{1}$, and the method of iterated regularity as stated in Proposition 3.4 , it is possible to show, after some easy but tedious computations (see [Ma] and $\S 5)$, that, microlocally near $L$, expressions of the form (2.14) belong to the space $I^{p, l}\left(\Lambda_{1}, \Lambda_{\pi(L)}\right)$ for some $p$ and $l$.

The above analysis is taking place in $G$ rather than in $\Gamma \backslash G$, and one still needs to push-forward these Lagrangians down to $\Gamma \backslash G$. Some technical difficulties may arise in doing so. For the case considered in Example 2.15, it is possible to show that the push-forward of the Lagrangian manifolds is an embedded manifold only for $t$ rational. For other values of $t$, the push-forward is a nonclosed manifold. However, proving isospectrality for $t$ in a dense set would yield the same result for all $t$. With this in mind, we formulate

Proposition 3.16. (1) Under assumptions (3.9) and (3.11), the push-forward of $\Lambda_{1} \subset T^{*}(G \times G)$ by the canonical projection is a closed submanifold of $T^{*}(\Gamma \backslash G \times \Gamma \backslash G)$.

(2) If there is a basis of $G$ in which $B_{i j}^{l} \in \mathbb{Q}$ and in which the entries of the matrix representing $\phi$ are also rational then for $t$ in a dense set of $\mathbb{R}$, the push-forward of $\Lambda_{0}$ is a closed manifold of $T^{*}(\Gamma \backslash G \times \Gamma \backslash G)$.

Proof. Let $\pi: G \times G \rightarrow \Gamma \backslash G \times \Gamma \backslash G$ denote the canonical projection. From the proof of Proposition 3.12 we see that $\Lambda_{1}$ is the conormal bundle to the submanifold $S_{1} \subset G \times G$ given by equation (3.15). Thus it is enough to show that $\pi\left(S_{1}\right)$ is a closed manifold of $\Gamma \backslash G \times \Gamma \backslash G$.

Let $\sigma \in \Gamma$ and consider the pointin $S_{1},(x+\sigma, z+c+B(\sigma, x) ; y, w)$, 
where $(y, w)$ satisfies

$$
\begin{aligned}
& y_{i}=x_{i}+\sigma_{i}, \quad i=1, \ldots, n-k, \\
& w_{h}=z_{h}+c_{h}+B^{h}(\sigma, x)-B^{h}(x+\sigma, x+\sigma-y), \quad h \notin\left\{1, p_{n-k}, \ldots, p_{n}\right\}, \\
& w_{1}=z_{1}+c_{1}+B^{1}(\sigma, x)-B^{1}(x+\sigma, x+\sigma-y) \\
& -\frac{1}{t} \sum_{h=n-k+1}^{n} \frac{x_{h}+\sigma_{h}-y_{h}}{b_{h}}\left(z_{p_{h}}+c_{p_{h}}+B^{p_{h}}(\sigma, x)\right. \\
& \left.-w_{p_{h}}-B^{p_{h}}(x+\sigma, x+\sigma-y)\right) . \\
& u_{i}=x_{i}, \quad i=1, \ldots, n-k \text {, } \\
& u_{i}=y_{i}-\sigma_{i}, \quad i=n-k+1, \ldots, n \text {, } \\
& v_{p_{h}}=w_{p_{h}}-c_{p_{h}}-B^{p_{h}}(\sigma, \sigma)-B^{p_{h}}(\sigma, x), \quad h=n-k+1, \ldots, n, \\
& v_{h}=z_{h}+B^{h}(\sigma, \sigma)+B^{h}(\sigma, x)-\sum_{i=1}^{n} \sum_{j=n-k+1}^{n} B_{i j}^{h}\left(x_{i}+\sigma_{i}\right)\left(x_{j}+\sigma_{j}-y_{j}\right) \\
& -\sum_{i=1}^{n} \sum_{j=1}^{n-k} B_{i j}^{h} \sigma_{i}\left(x_{j}+\sigma_{j}\right)-\sum_{i=1}^{n} \sum_{j=n-k+1}^{n} B_{i j}^{h} \sigma_{i} y_{j}, \quad h \notin\left\{1, p_{n-k}, \ldots, p_{n}\right\}, \\
& v_{1}=z_{1}+B^{1}(\sigma, x)+B^{1}(\sigma, \sigma) \\
& -\frac{1}{t} \sum_{h=n-k+1}^{n} \frac{x_{h}+\sigma_{h}-y_{h}}{b_{h}}\left(z_{p_{h}}+c_{p_{h}}+B^{p_{h}}(\sigma, x)\right. \\
& \left.-w_{p_{h}}-B^{p_{h}}(x+\sigma, x+\sigma-y)\right) \\
& -\sum_{i=1}^{n} \sum_{j=n-k+1}^{n} B_{i j}^{1}\left(x_{i}+\sigma_{i}\right)\left(x_{j}+\sigma_{j}-y_{j}\right) \\
& -\sum_{i=1}^{n} \sum_{j=1}^{n-k} B_{i j}^{1} \sigma_{i}\left(x_{j}+\sigma_{j}\right)-\sum_{i=1}^{n} \sum_{j=n-k+1}^{n} B_{i j}^{1} \sigma_{i} y_{j} .
\end{aligned}
$$

After some cancellations, this is the same as the point $(x+\sigma, z+c+B(\sigma, x)$; $u, v)$, where

$$
\begin{aligned}
u_{i} & =x_{i}, \quad i=1, \ldots, n-k, \\
u_{i} & =y_{i}-\sigma_{i}, \quad i=n-k+1, \ldots, n, \\
v_{p_{h}} & =w_{p_{h}}-c_{p_{h}}-B^{p_{h}}(\sigma, \sigma)-B^{p_{h}}(\sigma, x), \quad h=n-k+1, \ldots, n, \\
v_{h} & =z_{h}-\sum_{i=1}^{n} \sum_{j=n-k+1}^{n} B_{i j}^{h} x_{i}\left(x_{j}+\sigma_{j}-y_{j}\right), \quad h \notin\left\{1, p_{n-k}, \ldots, p_{n}\right\}, \\
v_{1}= & z_{1}-\frac{1}{t} \sum_{h=n-k+1}^{n} \frac{x_{h}+\sigma_{h}-y_{h}}{b_{h}}\left(z_{p_{h}}+c_{p_{h}}+B^{p_{h}}(\sigma, x)-w_{p_{h}}\right. \\
& -\sum_{i=1}^{n} \sum_{j=n-k+1}^{n} B_{i j}^{1} x_{i}\left(x_{j}+\sigma_{j}-y_{j}\right),
\end{aligned}
$$


which may be written as $(x+\sigma, z+c+B(\sigma, x) ; u, v)$, where now

$$
\begin{aligned}
u_{i} & =x_{i}, \quad i=1, \ldots, n-k, \\
v_{h} & =z_{h}-\sum_{i=1}^{n} \sum_{j=n-k+1}^{n} B_{i j}^{h} x_{i}\left(x_{j}-u_{j}\right), \quad h \notin\left\{1, p_{n-k}, \ldots, p_{n}\right\}, \\
v_{1} & =z_{1}-\frac{1}{t} \sum_{h=n-k+1}^{n} \frac{x_{h}-u_{h}}{b_{h}}\left(z_{p_{h}}-v_{p_{h}}-B^{p_{h}}(x, x-u)\right) \\
& -\sum_{i=1}^{n} \sum_{j=n-k+1}^{n} B_{i j}^{1} x_{i}\left(x_{j}-u_{j}\right) .
\end{aligned}
$$

Multiplying now $(x+\sigma, z+c+B(\sigma, x))$ on the left by $(-\sigma, B(\sigma, \sigma)-c)$, we obtain the point $(x, z ; u, v)$, with

$$
\begin{aligned}
& u_{i}=x_{i}, \quad i=1, \ldots, n-k, \\
& v_{h}=z_{h}-B^{h}(x, x-u), \quad h \notin\left\{1, p_{n-k}, \ldots, p_{n}\right\}, \\
& v_{1}=z_{1}-\frac{1}{t} \sum_{h=n-k+1}^{n} \frac{x_{h}-u_{h}}{b_{h}}\left(z_{p_{h}}-v_{p_{h}}-B^{p_{h}}(x, x-u)\right)-B^{1}(x, x-u)
\end{aligned}
$$

which also lays in $S_{1}$. We note that it is also possible to write $S_{1}$ as

$$
\begin{aligned}
S_{1}=\left\{(x, z ; y, w) \in G \times G: x_{i}=y_{i}, i=1, \ldots, n-k\right. \\
z_{h}=w_{h}-B^{h}(x, x-y), h \notin\left\{1, p_{n-k}, \ldots, p_{n}\right\} \\
\left.z_{1}=w_{1}-\frac{1}{t} \sum_{h=n-k+1}^{n} \frac{x_{h}-y_{h}}{b_{h}}\left(z_{p_{h}}-w_{p_{h}}-B^{p_{h}}(x, x-y)\right)-B^{1}(x, x-y)\right\},
\end{aligned}
$$

so a similar argument applies to a point in $S_{1}$ of the form $(x, z ; y+\sigma, w+$ $c+B(\sigma, y))$. Hence, $S_{1}$ is invariant under the action of $\Gamma$. Thus $\pi\left(S_{1}\right)$ is a closed submanifold of $\Gamma \backslash G$.

With respect to the second part of the proposition, we will prove that, for $t \in \mathbb{Q}, \pi\left(\operatorname{Gr}\left(\phi_{t}\right)\right)=\left\{\left(\pi(g), \pi\left(\phi_{t}(g)\right)\right): g \in G\right\}$ is a closed submanifold of $\Gamma \backslash G \times \Gamma \backslash G$. Without loss of generality (i.e., by scaling) we may assume that we have chosen a basis in which the constants of structure are integers, and the lattice $\Gamma \subset \mathbb{Z}^{m+n}$.

If, in this basis, the matrix of $\phi_{t}(x)$ has rational entries and $t$ is rational, then there is $m \in \mathbb{Z}$, such that $m \phi_{t}(x)$ has integer coefficients. Thus, there exist sublattices $\Gamma_{1}, \Gamma_{2} \triangleleft \Gamma$, with the inclusions of finite index and such that $\phi_{t}(x)$ induces a diffeomorphism $\hat{\phi}_{t}: \Gamma_{1} \backslash G \rightarrow \Gamma_{2} \backslash G$. Let $\pi_{i}: \Gamma_{i} \backslash G \rightarrow \Gamma \backslash G, i=1,2$, denote the canonical projection.

Since $\Gamma_{i} \triangleleft \Gamma$, have finite index, the maps $\pi_{i}, i=1,2$, are proper and we see that the set $\left\{\left(\pi_{1}(g), \pi_{2}\left(\hat{\phi}_{t}(g)\right)\right): g \in \Gamma_{1} \backslash G\right\}$ is a closed submanifold of $\Gamma \backslash G \times \Gamma \backslash G$. But $\left\{\left(\pi(g), \pi\left(\phi_{t}(g)\right)\right): g \in G\right\}=\left\{\left(\pi_{1}(g), \pi_{2}\left(\hat{\phi}_{t}(g)\right)\right): g \in \Gamma_{1} \backslash G\right\}$. Hence, for rational $t, \pi\left(\operatorname{Gr}\left(\phi_{t}\right)\right)$ is a closed submanifold of $\Gamma \backslash G \times \Gamma \backslash G$ and the proposition follows. 
Recall that $\Lambda_{2}=\Lambda_{\pi(L)}$, is the flow-out from $\pi(L)$, where $L$ is a submanifold of $\Lambda_{0} \cap \Lambda_{1}$. It foilows that under the same hypotheses as in Proposition 3.16, the push-forward, $\pi_{*}\left(\Lambda_{2}\right)$, is also a closed submanifold of $\Gamma \backslash G \times \Gamma \backslash G$.

Putting together Propositions 2.12, 3.12, and 3.16, we obtain

Theorem 3.17. Under assumptions (3.9) and (3.11), and if there is a basis of $G$ in which $B_{i j}^{l} \in \mathbb{Q}$ and in which the entries representing $\phi$ are also rational, then, for each $t \in \mathbb{Q}$, the operators $S_{t}$ have a Schwartz kernel $K(x, z, y, w)$ of the form given by equation (2.14), where the phase is given by (2.10). Microlocally, the kernels of these operators satisfy

$$
K(x, z, y, w) \in \sum_{\substack{i, j \in\{0,1,2\} \\ i<j}} I^{p_{i j}, q_{i j}}\left(\Lambda_{i}, \Lambda_{j}\right),
$$

for some $p_{i j}, q_{i j} \in \mathbb{R}$.

\section{THE GEOMETRY OF BLOW-DOWN MAPPINGS}

Motivated by the above examples, and with the goal of developing a functional calculus for these classes of operators in mind, we formulate a class of canonical relations incorporating the features speiled out in Proposition 3.12 and Theorem 3.17.

Consider two manifolds $X^{n}$ and $Y^{n}$ and a mapping $f: X \rightarrow Y$. Let $\omega$ be a volume form on $Y$. We are interested in the following kind of singularity of the map $f$.

Definition 4.1 [Gu1]. A smooth mapping $f: X \rightarrow Y$ is a blow-down map along a smooth hypersurface, $L$, if $f$ is a local diffeomorphism away from $L$, while along $L, f$ has constant rank $n-k$ so at $L, d f_{s}$ drops rank by $k \leq n$. Further, the kernel of $d f_{s}$ is contained in $T_{s} L$ and $f^{*} \omega$ vanishes exactly to $k$ th order on $L$

Note that the requirement that $f^{*} \omega$ vanishes exactly to $k$ th order on $L$ means that the determinant of the Jacobian matrix of $f$ vanishes to $k$ th order at $L$ and this condition is independent of $\omega$. One has

Proposition 4.2 [Gu1]. If $f$ is a blow-down along $L$ then at every point $m_{0} \in L$ one can find a local system of coordinates $\left(x_{1}, \ldots, x_{n}\right)$ centered at $m_{0}$ and $a$ local system of coordinates $\left(y_{1}, \ldots, y_{n}\right)$ centered at $f\left(m_{0}\right)$ so that $f^{*}\left(y_{i}\right)=$ $x_{i}, i=1, \ldots, n-k$, and $f^{*}\left(y_{i}\right)=x_{i} x_{1}, i=n-k+1, \ldots, n$.

If the target manifold is endowed with a symplectic form, the above structure of $f$ can be made compatible with a set of canonical symplectic coordinates.

Proposition 4.3. Let $\pi: C \rightarrow X^{2 n}$ be a blow-down along $L \subset C$, where it drops rank by $1 \leq k<n$. Assume $X$ is symplectic and $\pi(L)$ is involutive. Let $m_{0} \in L$. Then there are local coordinates $t$ on $C$ and local canonical coordinates $(x, \xi)$ on $X$ so that $\pi^{*}\left(x_{i}\right)=t_{i}, i=1, \ldots, n ; \pi^{*}\left(\xi_{i}\right)=t_{n+i}, i=1, \ldots, n-k$; $\pi^{*}\left(\xi_{i}\right)=t_{n+1} t_{n+i}, i=n-k+1, \ldots, n$.

Proof. Let $\left(\bar{t}_{1}, \ldots, \bar{t}_{2 n}\right)$ (resp. $\left.\left(s_{1}, \ldots, s_{2 n}\right)\right)$ be a coordinate system around $m_{0}$ (resp. $\pi\left(m_{0}\right)$ ) as in Proposition 4.2, then $L=\left\{\bar{t}_{1}=0\right\}$ and $S=\pi(L)=$ $\left\{s_{1}=s_{2 n-k+1}=\cdots=s_{2 n}=0\right\}$. Since $S$ is involutive, the Hamiltonian vector 
field $H_{S_{1}}$ is tangent to $S$. Choose a $(2 n-1)$-dimensional manifold $W_{1} \subset X$ containing $\pi\left(m_{0}\right)$ and transversal to $H_{s_{1}}$ and choose a function $a$, defined on $W_{1}$, so that $\left.a\right|_{W_{1} \cap S}=0$ but $\left.d a\right|_{\pi\left(m_{0}\right)}$ and $\left.d s_{1}\right|_{\pi\left(m_{0}\right)}$ are linearly independent at $T_{\pi\left(m_{0}\right)} W_{1}$. Note that $\operatorname{dim}\left(T_{\pi\left(m_{0}\right)} W_{1}\right)=2 n-1$, whereas $\operatorname{dim}\left(S \cap W_{1}\right)=2 n-k-2$, so the codimension of $S \cap W_{1}$ in $W_{1}$ is $k+1$. We may impose the conditions

$$
\left.d a\right|_{T_{\pi\left(m_{0}\right)}\left(S \cap W_{1}\right)}=\left.d s_{1}\right|_{T_{\pi\left(m_{0}\right)}\left(S \cap W_{1}\right)}=0
$$

and $d a, d s_{1}$ are linearly independent on the complement of $T_{\pi\left(m_{0}\right)}\left(S \cap W_{1}\right)$ in $T_{\pi\left(m_{0}\right)} W_{1}$, which must be $k+1$ dimensional.

We can extend the function $a$ to a neighborhood of $\pi\left(m_{0}\right) \in Y$ by requiring that be constant along the flow of $H_{s_{1}}$. Let $\xi_{1}=s_{1}, \xi_{n-k+1}=a$. We have, $H_{\xi_{1}} \xi_{n-k+1}=0$, since $\xi_{n-k+1}$ is constant along the integral curves of $\xi_{1}$. Also, $\xi_{n-k+1} \mid s=0$ (so $H_{\xi_{n-k+1}}$ is also tangent to $S$ at $m_{0}$ ) and $d \xi_{1} \wedge d \xi_{n-k+1} \neq 0$ at $m_{0}$ and therefore does not vanish in a small neighborhood of $m_{0}$.

Pick a submanifold $W_{2}$ transverse to both $H_{\xi_{1}}$ and $H_{\xi_{n-k+1}}$. Necessarily, $\operatorname{dim}\left(W_{2}\right)=2 n-2$ and $\operatorname{dim}\left(W_{2} \cap S\right)=2 n-k-3$ so $\left(S \cap W_{2}\right)$ has codimension $k+1$ in $W_{2}$. We may thus pick $\xi_{n-k+2}$, defined on $W_{2}$, so that $\left.\xi_{n-k+2}\right|_{S \cap W_{2}}=0$ and $d \xi_{n-k+2}$ is linearly independent of $d \xi_{1}$ and $d \xi_{n-k+1}$ at $T_{\pi\left(m_{0}\right)}$.

Extend, as above, $\xi_{n-k+2}$ to be constant along the integral surfaces of $H_{\xi_{1}}$, and $H_{\xi_{n-k+1}}$. (The latter form an integrable distribution, since the Lie bracket $\left.\left\{H_{\xi_{1}}, H_{\xi_{n-k+1}}\right\}=H_{\left\{\xi_{1}, \xi_{n-k+1}\right\}}=H_{H_{\xi_{1}} \xi_{n-k+1}}=0\right)$. Now, we have that $H_{\xi_{1}} \xi_{n-k+2}=$ $H_{\xi_{n-k+1}} \xi_{n-k+2}=0, \xi_{n-k+2} \mid s=0$, and $d \xi_{1}, d \xi_{n-k+1}, d \xi_{n-k+2}$ are linearly independent in a neighborhood of $\pi\left(m_{0}\right)$.

We can continue this procedure to find $\xi_{1}, \xi_{n-k+1}, \ldots, \xi_{n}$, satisfying

$$
\begin{aligned}
& H_{\xi_{i}} \xi_{j}=0, \quad i, j=1, n-k+1, \ldots, n, \\
& d \xi_{1} d \xi_{n-k+1}, \ldots, d \xi_{n} \text { are linearly independent at } m, \\
& \left.\xi_{1}\right|_{S}=\left.\xi_{n-k+1}\right|_{S}=\cdots=\left.\xi_{n}\right|_{s}=0
\end{aligned}
$$

in a neighborhood of $m_{0}$. Since $H_{\xi_{i}} \xi_{j}=0$, the vector fields $H_{\xi_{1}}, H_{\xi_{n-k+1}}, \ldots$, $H_{\xi_{n}}$ form an integrable distribution. Complete $\xi_{1}, \xi_{n-k+1}, \ldots, \xi_{n}$ to form a canonical coordinate system on $X$, say $(x, \xi)$. By construction $\left.\xi_{1}\right|_{s}=$ $\xi_{n-k+1}\left|s=\cdots=\xi_{n}\right|_{S}=0$, so for dimensional reasons, $S=\left\{\xi_{1}=\xi_{n-k+1}=\right.$ $\left.\cdots=\xi_{n}=0\right\}$ around a small neighborhood of $\pi\left(m_{0}\right)$.

Now, we have $\bar{t}_{1}=\xi_{1} \circ \pi$, by construction, and $0=\left.\xi_{1} \circ \pi\right|_{\left\{\bar{t}_{1}=0\right\}}=\xi_{n-k+1} \circ$ $\left.\pi\right|_{\left\{\bar{t}_{1}=0\right\}}=\cdots=\left.\xi_{n} \circ \pi\right|_{\left\{\bar{t}_{1}=0\right\}}=0$. Thus, there are functions $f_{n-k+1}(\bar{t}), \ldots, f_{n}(\bar{t})$ so that $\xi_{i} \circ \pi=\bar{t}_{1} f_{i}(\bar{t}), i=n-k+1, \ldots, n$, and $\pi(\bar{t})=\left(\bar{t}_{1}, G(\bar{t}), \bar{t}_{1} f_{n-k+1}(\bar{t})\right.$, $\left.\ldots, \bar{t}_{1} f_{n}(\bar{t})\right)$ where $G: R^{2 n} \rightarrow R^{2 n-k-1}$. In these coordinates,

$$
\left.d \pi\right|_{\left\{\bar{t}_{1}=0\right\}}=\left(\begin{array}{cccc}
1 & 0 & \ldots & 0 \\
& \left(\left.D G\right|_{\tilde{t}_{1}=0}\right)_{(2 n-k-1) \times 2 n} & & \\
f_{n-k+1} & 0 & \ldots & 0 \\
f_{n-k+2} & 0 & \ldots & 0 \\
\vdots & \vdots & \ddots & \vdots \\
f_{n} & 0 & \ldots & 0
\end{array}\right) .
$$

Since $\left.d \pi\right|_{\left\{t_{1}=0\right\}}$ has rank $2 n-k$, then $\left.D G\right|_{\left\{\tilde{t}_{1}=0\right\}}$ must have rank $2 n-k-1$. Also, $d \pi$ has full rank away from $\left\{\bar{t}_{1}=0\right\}$, so $\bar{t}_{1}, D G_{1}, \ldots, D G_{2 n-k-1}$ are linearly independent at every point. Set $\tilde{t}_{n+1}=\bar{t}_{1}, \tilde{t}_{i}=G_{i}, i=1, \ldots, n$; 
$\tilde{t}_{i}=G_{i}, i=n+2, \ldots, 2 n-k-1$, and complete $\left(\tilde{t}_{1}, \tilde{t}_{n+1}, \ldots, \tilde{t}_{2 n-k}\right)$ to form a coordinate system $\left(\tilde{t}_{1}, \ldots, \tilde{t}_{2 n}\right)$ on $X$. Now, $L$ is defined locally by $\tilde{t}_{n+1}=0$.

As before we obtain functions $g_{n-k+1}(t), \ldots, g_{n}(t)$ so that $\xi_{i} \circ \pi=\tilde{t}_{n+1} g_{i}(\tilde{t})$, $i=n-k+1, \ldots, n$, and $\pi(t)=\left(\tilde{t}_{1}, \ldots, \tilde{t}_{2 n-k}, \tilde{t}_{n+1} g_{k+1}(t), \ldots, \tilde{t}_{n+1} g_{n}\right)$. From the fact that the rank of $d \pi$ is $2 n-k$ on $L$, we see that $g_{i} \neq 0$ on $L$. Further, the Jacobian determinant of $f$ in these coordinates is

$$
\tilde{t}_{n+1}^{k} \operatorname{det}\left(\frac{\partial g_{i}}{\partial \tilde{t}_{j}}\right), \quad k+1 \leq i \leq n, 2 n-k+1 \leq j \leq 2 n .
$$

Since $d \pi$ vanishes at $L$ exactly to order $k$, the determinant of the matrix $\left(\partial g_{i} / \partial \tilde{t}_{j}\right)$ cannot be zero, for, otherwise $f^{*} \omega$ would vanish to order higher than $k$. Hence we obtain the desired form by making the change of variables

$$
\begin{gathered}
t_{i}=\tilde{t}_{i}, \quad i=1, \ldots, 2 n-k, \\
t_{i}=g_{i}, \quad i=2 n-k+1, \ldots, 2 n .
\end{gathered}
$$

Let $X$ and $Y$ be two $n$-dimensional manifolds. From now on we let $C \subset T^{*}(X) \times T^{*}(Y)$ denote a homogeneous canonical relation satisfying the following: away from a hypersurface $L \subset C$, both projections $\pi: C \rightarrow T^{*}(X)$ and $\rho: C \rightarrow T^{*}(Y)$ are diffeomorphisms, whereas at $L$ they are both blowdowns dropping rank by $k<n$ and $\pi(L), \rho(L)$ are nonradial, involutive submanifolds of codimension $k+1$.

For a symplectic vector space, $W$, and subspace, $A \subset W$, we let $A^{\sigma}$ denote the orthogonal complement of $A$ with respect to the symplectic form on $W$.

Lemma 4.5 [Gu1]. Let $m \in L \subset C$ then at $T_{m} C$ :

(1) $d \rho(\operatorname{ker} d \pi)=(\operatorname{Im} d \rho)^{\sigma}$,

(2) $d \pi(\operatorname{ker} d \rho)=(\operatorname{Im} \pi)^{\sigma}$.

Proof. Let $U \in \operatorname{ker} d \pi$. Since $C$ is Lagrangian, for all $W \in T_{m} C$, we have $\omega_{T^{*} Y}(d \rho(U), d \rho(W))=\omega_{T^{*} X}(d \pi(U), d \pi(W))=0$, so $d \rho(X) \in(\operatorname{Im} d \rho)^{\sigma}$. Hence, $d \rho(\operatorname{ker}(d \pi)) \subset(\operatorname{Im} d \rho)^{\sigma}$. But, since $\operatorname{ker} d \pi$ and $\operatorname{ker} d \rho$ are transverse (for considering $T_{m} C \subset T_{m}\left(T^{*}(X \times Y)\right)=T_{\pi(m)}\left(T^{*} X\right) \oplus T_{\rho(m)}\left(T^{*} Y\right)$, we have that $\operatorname{ker} d \pi \subset T_{\rho(m)}\left(T^{*} Y\right)$ and $\left.\operatorname{ker} d \rho \subset T_{\pi(m)}\left(T^{*} X\right)\right)$ and both have the same dimension, $\operatorname{dim}(d \rho(\operatorname{ker} d \pi))=\operatorname{dim}(\operatorname{Im} d \rho)^{\sigma}$. Hence (1) follows. The proof of (2) is completely analogous.

Even though the projections $\rho$ and $\pi$ cannot both be conjugated simultaneously to a normal form as in 4.3 , we can still parametrize the lagrangian manifold $C \subset T^{*} X \times Y$ by means of a phase function satisfying certain restrictions. We will do this next, but first we need some notation. Since we are going to work locally at a point $m \in L$, we will assume $X=Y=\mathbb{R}^{n}$.

For points $x \in \mathbb{R}^{n}$ we will denote, from now on, $x=\left(x^{\prime}, x^{\prime \prime}\right) \in \mathbb{R}^{n-k} \times \mathbb{R}^{k}$. Let $m \in L \subset C$. By Proposition 4.3 we can find local coordinates $(s, \sigma),(t, \tau)$ in $C$ and local canonical coordinates $(x ; \xi)$ in $T^{*}(X),(y, \eta)$ in $T^{*}(Y)$ so that in a neighborhood of $m \in L, \pi(s, \sigma)=\left(s ; \sigma^{\prime}, \sigma_{1} \sigma_{n-k+1}, \ldots, \sigma_{1} \sigma_{n}\right)$, 
$\rho(t, \tau)=\left(t, \tau^{\prime} ; \tau_{1} \tau_{n-k+1}, \tau_{1} \tau_{n}\right)$. Let us change coordinates on $C$ as follows:

$$
\begin{aligned}
\sigma_{i} & =s_{i}-T_{i}, \quad i=n-k+1, \ldots, n, \\
\tau_{i} & =S_{i}-t_{i}, \quad i=n-k+1, \ldots, n, \\
t_{1} & =S_{1}+\frac{1}{2}, \sum_{i=n-k+1}^{n}\left(S_{i}-t_{i}\right)^{2} .
\end{aligned}
$$

Then in a neighborhood of $m$,

$$
L=\left\{\sigma_{1}=0\right\}=\left\{\tau_{1}=0\right\},
$$

and in these coordinates,

$$
\begin{gathered}
\pi\left(s ; \sigma^{\prime}, T^{\prime \prime}\right)=\left(s ; \sigma^{\prime}, \sigma_{1}\left(s^{\prime \prime}-T^{\prime \prime}\right)\right), \\
\rho\left(S_{1}, t_{2}, \ldots, t_{n}, \tau^{\prime}, S^{\prime \prime}\right) \\
=\left(S_{1}+\frac{1}{2} \sum_{i=n-k+1}^{n}\left(S_{i}-t_{i}\right)^{2}, t_{2}, \ldots, t_{n} ; \tau^{\prime}, \tau_{1}\left(S^{\prime \prime}-t^{\prime \prime}\right)\right) .
\end{gathered}
$$

Let $\left\langle\left\{e_{i}\right\}_{i=1}^{n}\right\rangle$ denote the linear span formed by the vectors $\left\{e_{i}\right\}_{i=1}^{n}$. Then

$$
\begin{aligned}
\left.\operatorname{ker} d \pi\right|_{L} & =\left\langle\left\{\frac{\partial}{\partial T_{i}}\right\}_{i=n-k+1}^{n}\right\rangle, \\
\operatorname{ker} d \rho & =\left\langle\left\{\left(S_{i}-t_{i}\right) \frac{\partial}{\partial S_{1}}-\frac{\partial}{\partial S_{i}}\right\}_{i=n-k+1}^{n}\right\rangle .
\end{aligned}
$$

We also compute the pullback, $\omega_{C}$, of the symplectic form $\omega_{T^{*} X}$, in $T^{*} X$ as follows:

$$
\begin{aligned}
\omega_{C}= & \pi^{*} \omega_{T^{*} X}=d \sigma_{1} \wedge\left(d s_{1}+\sum_{i=n-k+1}^{n}\left(s_{i}-T_{i}\right) d s_{i}\right) \\
& +\sum_{i=2}^{n-k} d \sigma_{i} \wedge d s_{i}+\sigma_{1} \sum_{i=n-k+1}^{n}\left(d s_{i} \wedge d T_{i}\right) .
\end{aligned}
$$

Since $C$ is Lagrangian this is equal to

$$
\begin{aligned}
\rho^{*} \omega_{T^{*} Y}= & d \tau_{1} \wedge\left(d S_{1}+\sum_{i=n-k+1}^{n}\left(S_{i}-t_{i}\right) d S_{i}\right) \\
& +\sum_{i=2}^{n-k} d \tau_{i} \wedge d t_{i}+\tau_{1} \sum_{i=n-k+1}^{n}\left(d S_{i} \wedge d t_{i}\right) .
\end{aligned}
$$

At points of $L$,

$$
\begin{aligned}
\left.\omega_{C}\right|_{L} & =d \sigma_{1} \wedge\left(d s_{1}+\sum_{i=n-k+1}^{n}\left(s_{i}-T_{i}\right) d s_{i}\right)+\sum_{i=2}^{n-k} d \sigma_{i} \wedge d s_{i} \\
& =d \tau_{1} \wedge\left(d S_{1}+\sum_{i=n-k+1}^{n}\left(S_{i}-t_{i}\right) d S_{i}\right)+\sum_{i=2}^{n-k} d \tau_{i} \wedge d S_{i}
\end{aligned}
$$


So

$$
\begin{aligned}
\operatorname{ker}\left(\left.\omega_{C}\right|_{L}\right) & =\left\langle\left\{\frac{\partial}{\partial T_{i}},\left(s_{i}-T_{i}\right) \frac{\partial}{\partial s_{1}}-\frac{\partial}{\partial s_{i}}\right\}_{i=n-k+1}^{n}\right\rangle \\
& =\left\langle\left\{\frac{\partial}{\partial t_{i}},\left(S_{i}-t_{i}\right) \frac{\partial}{\partial S_{1}}-\frac{\partial}{\partial S_{i}}\right\}_{i=n-k+1}^{n}\right\rangle
\end{aligned}
$$

Let $m=\left(S_{1}, t_{2}, \ldots, t_{n}, \tau^{\prime}, S^{\prime \prime}\right) \in L$ and consider $\left.d \rho\right|_{m}: T_{m} C \rightarrow T_{\rho(m)}\left(T^{*} Y\right)$. We have

$$
\begin{aligned}
\left.\operatorname{Im} d \rho\right|_{m}=\langle & \frac{\partial}{\partial y_{1}}, \ldots, \frac{\partial}{\partial y_{n-k}}, \\
\frac{\partial}{\partial y_{n-k+1}}-\left(S_{n-k+1}-t_{n-k+1}\right) \frac{\partial}{\partial y_{1}}, \ldots, \frac{\partial}{\partial y_{n}}-\left(S_{n}-t_{n}\right) \frac{\partial}{\partial y_{1}} & \left.\left.\frac{\partial}{\partial \eta_{1}}+\sum_{i=n-k+1}^{n}\left(S_{i}-t_{i}\right) \frac{\partial}{\partial \eta_{i}}, \frac{\partial}{\partial \eta_{2}}, \ldots, \frac{\partial}{\partial \eta_{n-k}}\right\}\right\rangle ;
\end{aligned}
$$

thus, the orthogonal complement of $\left.\operatorname{Im} d \rho\right|_{m}$ in $T_{\rho(m)}\left(T^{*} Y\right)$ is

$$
\begin{aligned}
\left(\left.\operatorname{Im} d \rho\right|_{m}\right)^{\sigma} & =\left\langle\left\{\left(S_{i}-t_{i}\right) \frac{\partial}{\partial y_{1}}-\frac{\partial}{\partial y_{i}}\right\}_{i=n-k+1}^{n}\right\rangle \\
& =\left\langle\left\{\left.d \rho\right|_{m}\left(\frac{\partial}{\partial t_{n-k+1}}\right), \ldots,\left.d \rho\right|_{m}\left(\frac{\partial}{\partial t_{n}}\right)\right\}\right\rangle .
\end{aligned}
$$

By Lemma 4.5 and the fact that $\operatorname{ker}(d \rho)$ and $\operatorname{ker}(d \pi)$ are transversal at every point, we have that

$$
\left\langle\left\{\frac{\partial}{\partial t_{n-k+1}}, \ldots, \frac{\partial}{\partial t_{n}}\right\}\right\rangle=\left.\operatorname{ker} d \pi\right|_{m}=\left\langle\left\{\frac{\partial}{\partial T_{n-k+1}}, \ldots, \frac{\partial}{\partial T_{n}}\right\}\right\rangle
$$

and the 1 -forms $d s_{i}, i=1, \ldots, n, d t_{i}, i=n-k+1, \ldots, n$, and $d \tau_{i}, i=$ $1, \ldots, n-k$, are linearly independent. Hence, about $m \in L$ we can use coordinates $s=\pi^{*} x, t_{i}=\rho^{*} y_{i}, i=n-k+1, \ldots, n$, and $\tau_{i}=\rho^{*} \eta_{i}, i=$ $1, \ldots, n-k$.

Thus $\left(x, \eta^{\prime}, y^{\prime \prime}\right)$ form a coordinate system on $C$ about $m \in L$. By [Hö1] there exists a generating function of the form $S\left(x, y^{\prime \prime}, \theta\right)$ for $L$, homogeneous of degree 1 in $\theta \in \mathbb{R}^{n-k}$. In this coordinate system,

$$
C=\left\{(x, \xi ; y, \eta): \xi=d_{x} S, y^{\prime}=d_{\theta} S, \eta^{\prime}=\theta, \eta^{\prime \prime}=d_{y^{\prime \prime}} S\right\}
$$

with $\left(x, y^{\prime \prime}, \theta\right) \in W$, a conic neighborhood of $\theta_{1}=0$ near $m$. Thus the phase function $\phi(x, y, \theta)=S\left(x, y^{\prime \prime}, \theta\right)-y^{\prime} \theta$ parametrizes $C$ near $m$.

The properties of $C$ translate into some restrictions on the generating function $S$ which we spell out next.

Since $\pi(L)=\left\{\xi_{1}=\xi_{n-k+1}=\cdots=\xi_{n}=0\right\}$ at $\theta_{1}=0$, the function $S\left(x, y^{\prime \prime}, 0, \theta_{2}, \ldots, \theta_{n-k}\right)$ does not depend on $x_{1}, x_{n-k+1}, \ldots, x_{n}$, i.e., $S\left(x, y^{\prime \prime}, 0, \theta_{2}, \ldots, \theta_{n-k}\right)=S_{1}\left(x_{2}, \ldots, x_{n-k}, y^{\prime \prime}, \theta_{2}, \ldots, \theta_{n-k}\right)$. Similarly, 
since $\rho(L)=\left\{\eta_{1}=\eta_{n-k+1}=\cdots=\eta_{n}=0\right\}$, we must have that $d_{y^{\prime \prime}} S_{1}=0$. Thus, we can write

$$
\begin{aligned}
S\left(x, y^{\prime \prime}, \theta\right) & =S\left(x, y^{\prime \prime}, 0, \theta_{2}, \ldots, \theta_{n-k}\right)+\theta_{1} S_{2}\left(x, y^{\prime \prime}, \theta\right) \\
& =S_{1}\left(x_{2}, \ldots, x_{n-k}, \theta_{2}, \ldots, \theta_{n-k}\right)+\theta_{1} S_{2}\left(x, y^{\prime \prime}, \theta\right),
\end{aligned}
$$

where $S_{1}$ is homogeneous of degree 1 in $\left(\theta_{2}, \ldots, \theta_{n-k}\right)$ and $S_{2}$ is homogeneous of degree 0 in $\theta$. Hence

$$
\begin{array}{r}
C=\left\{\left(x,\left(\theta_{1} \frac{\partial S_{2}}{\partial x_{1}}, \frac{\partial S}{\partial x_{2}}, \ldots, \frac{\partial S}{\partial x_{n-k}}, \theta_{1} d_{x^{\prime \prime}} S_{2}\right) ;\left(d_{\theta} S, y^{\prime \prime}\right),\left(\theta, \theta_{1} d_{y^{\prime \prime}} S_{2}\right)\right):\right. \\
\left.\left(x, y^{\prime \prime}, \theta\right) \in W\right\}
\end{array}
$$

and

$$
\begin{array}{r}
L=\left\{\left(x,\left(0, \frac{\partial S_{2}}{\partial x_{2}}, \ldots, \frac{\partial S_{2}}{\partial x_{n-k}}, 0\right) ;\left(d_{\theta} S, y^{\prime \prime}\right),\left(0, \theta_{2}, \ldots, \theta_{n-k}, 0\right)\right):\right. \\
\left.\left(x, y^{\prime \prime}, \theta_{2}, \ldots, \theta_{n-k}\right) \in W\right\} .
\end{array}
$$

The matrix representing $d \pi$ has the form

$$
d \pi=\left(\begin{array}{cccc}
I_{n \times n} & 0 & 0 & 0 \\
M & \theta_{1} N & \frac{\partial^{2} S}{\partial \theta_{1} \partial x_{1}} & \theta_{1} P \\
Q & \theta_{1} R & A & B \\
\theta_{1} T & \theta_{1} U & d_{x^{\prime \prime}}\left(\frac{\partial S}{\partial \theta_{1}}\right) & \theta_{1} V
\end{array}\right),
$$

where $M, N, P, Q, R, T, U$, and $V$ are submatrices of respective dimensions, $1 \times n, 1 \times k, 1 \times(n-k-1),(n-k-1) \times n,(n-k-1) \times k$, $k \times n, k \times k$, and $k \times(n-k-1) ; d_{x^{\prime \prime}}\left(\partial S / \partial \theta_{1}\right)$ is though of as a $k \times 1 \mathrm{ma}-$ trix and $A$ and $B$ are the matrices

$$
A=\left(\begin{array}{c}
\frac{\partial}{\partial \theta_{1}}\left(\frac{\theta_{1} \partial S_{2}}{\partial x_{2}}\right) \\
\vdots \\
\frac{\partial}{\partial \theta_{1}}\left(\frac{\dot{\theta}_{1} \partial S_{2}}{\partial x_{n-k}}\right)
\end{array}\right)_{(n-k-1) \times 1},
$$

and

So at $L$,

$$
B=\left[\frac{\partial^{2} S}{\partial x_{i} \partial \theta_{j}}\right]_{\substack{2 \leq i \leq n-k \\ 2 \leq j \leq n-k}}^{\substack{2 \leq j \\ \text {. }}}
$$

$$
\left.A\right|_{\left\{\theta_{1}=0\right\}}=\left(\begin{array}{c}
\frac{\partial S_{2}}{\partial x_{2}} \\
\vdots \\
\frac{\partial S_{2}}{\partial x_{n-k}}
\end{array}\right) \text { and }\left.B\right|_{\left\{\theta_{1}=0\right\}}=\left[\frac{\partial^{2} S_{1}}{\partial x_{i} \partial \theta_{j}}\right]_{\substack{2 \leq i \leq n-k \\
2 \leq j \leq n-k}} .
$$


Thus,

$$
\left.d \pi\right|_{L}=\left(\begin{array}{cccc}
I_{n \times n} & 0 & 0 & 0 \\
M & 0 & \frac{\partial S_{2}}{\partial x_{1}} & 0 \\
Q & 0 & \left.A\right|_{\left\{\theta_{1}=0\right\}} & \left.B\right|_{\left\{\theta_{1}=0\right\}} \\
0 & 0 & d_{x^{\prime \prime}} S_{2} & 0
\end{array}\right)
$$

But $\left.d \pi\right|_{L}$ has rank $2 n-k$, so $\operatorname{det}\left(\left.B\right|_{\left\{\theta_{1}=0\right\}}\right) \neq 0$, that is $S_{1}\left(x-2, \ldots, x_{n-k}\right.$, $\left.\theta_{2}, \ldots, \theta_{n-k}\right)$ is a nondegenerate phase function. Changing variables canonically in $T^{*} X$ we may assume that

$$
S_{1}\left(x_{2}, \ldots, x_{n-k}, \theta_{2}, \ldots, \theta_{n-k}\right)=\sum_{i=2}^{n-k} x_{i} \theta_{i} .
$$

The rate of vanishing of the canonical symplectic form on $L$ imposes that $\operatorname{det}(d \pi)$ vanishes at $L$ to order $k$; but

$$
\begin{aligned}
\operatorname{det}(d \pi) & =\operatorname{det}\left(\begin{array}{ccc}
\theta_{1} N & \frac{\partial^{2} S}{\partial \theta_{1} \partial x_{1}} & \theta_{1} P \\
\theta_{1} R & A & B \\
\theta_{1} U & d_{x^{\prime \prime}}\left(\frac{\partial S}{\partial \theta_{1}}\right) & \theta_{1} V
\end{array}\right) \\
& =\theta_{1}^{k} \operatorname{det}\left(\begin{array}{ccc}
N & \frac{\partial^{2} S}{\partial \theta_{1} \partial x_{1}} & \theta_{1} P \\
R & A & B \\
U & d_{x^{\prime \prime}}\left(\frac{\partial S}{\partial \theta_{1}}\right) & \theta_{1} V
\end{array}\right) \\
& =\theta_{1}^{k} \operatorname{det}\left(\begin{array}{ccc}
N & \frac{\partial^{2} S}{\partial \theta_{1} \partial x_{1}} & \theta_{1} P \\
U & d_{x^{\prime \prime}}\left(\frac{\partial S}{\partial \theta_{1}}\right) & \theta_{1} V \\
R & A & B
\end{array}\right) .
\end{aligned}
$$

Hence, near $L$,

$$
\begin{aligned}
0 \neq \operatorname{det} & \left(\begin{array}{ccc}
N & \frac{\partial^{2} S}{\partial \theta_{1} \partial x_{1}} \\
U & d_{x^{\prime \prime}}\left(\frac{\partial S}{\partial \theta_{1}}\right)
\end{array}\right) \\
& =\operatorname{det}\left(\begin{array}{cccc}
\frac{\partial^{2} S_{2}}{\partial y_{n-k+1} \partial x_{1}} & \cdots & \frac{\partial^{2} S_{2}}{\partial y_{n} \partial x_{1}} & \frac{\partial S_{2}}{\partial x_{1}}+\theta_{1} \frac{\partial^{2} S_{2}}{\partial \zeta_{1} \partial x_{1}} \\
\frac{\partial^{2} S_{2}}{\partial y_{n-k+1} \partial x_{n-k+1}} & \cdots & \frac{\partial^{2} S_{2}}{\partial y_{n} \partial x_{n-k+1}} & \frac{\partial S_{2}}{\partial x_{n-k+1}}+\theta_{1} \frac{\partial^{2} S_{2}}{\partial \zeta_{1} \partial x_{n-k+1}} \\
\vdots & \ddots & \vdots & \vdots \\
\frac{\partial^{2} S_{2}}{\partial y_{n-k+1} \partial x_{n}} & \cdots & \frac{\partial^{2} S_{2}}{\partial y_{n} \partial x_{n}} & \frac{\partial S_{2}}{\partial x_{n}}+\theta_{1} \frac{\partial^{2} S_{2}}{\partial \zeta_{1} \partial x_{n}}
\end{array}\right) .
\end{aligned}
$$

And, for similar reasons,

$$
\left(\frac{\partial S_{2}}{\partial x_{1}}, \frac{\partial S_{2}}{\partial x_{n-k+1}}, \ldots, \frac{\partial S_{2}}{\partial x_{n}}\right) \neq 0
$$

in a neighborhood of $m$ in $C$. Thus, we have 
Theorem 4.13. Assume that $C \subset T^{*}(X \backslash 0) \times T^{*}(Y \backslash 0)$ is a homogeneous canonical relation satisfying: away from a hypersurface $L \subset C$, both projections $\pi: C \rightarrow T^{*}(X)$ and $\rho: C \rightarrow T^{*}(Y)$ are diffeomorphisms, whereas at $L$ they are both blow-downs dropping rank by $k$ and both $\pi(L)$ and $\rho(L)$ are nonradial and involutive. Then there are canonical transformations $\chi_{1}: T^{*} R^{n} \backslash 0 \rightarrow T^{*} Y \backslash 0$ and $\chi_{2}: T^{*} X \backslash 0 \rightarrow T^{*} R^{n} \backslash 0$, such that $\operatorname{Gr}\left(\chi_{2}\right) \circ C \circ \operatorname{Gr}\left(\chi_{1}\right)$ is parametrized by a phase function of the form

$$
\phi(x, y, \theta)=\sum_{i=2}^{n-k}\left(x_{i}-y_{i}\right) \theta_{i}-y_{1} \theta_{1}+\theta_{1} S_{2}\left(x, y^{\prime \prime}, \theta\right)
$$

satisfying (4.11) and (4.12).

\section{ITERATED REgULARITY AND THE PROOF OF THEOREM 1.2}

Let $L \subset C \subset\left(T^{*} X \backslash 0\right) \times\left(T^{*} Y \backslash 0\right)$ as in the previous sections and consider the properly supported Fourier integral operators $A \in I^{m}(C), B \in I^{m^{\prime}}\left(C^{t}\right)$. By $\Lambda_{0}=\Lambda_{\pi(L)}$ we denote the flow-out of $\pi(L)$ in $\left(T^{*} X \backslash 0\right) \times\left(T^{*} X \backslash 0\right)$.

If $m, m^{\prime} \in\left(C \times C^{t}\right) \cap\left(T^{*} X \times \Delta_{T^{*} Y} \times T^{*} X\right)$, then $\rho(m)=\rho\left(m^{\prime}\right)$; so if $m$ or $m^{\prime}$ is in $C \backslash L$, then so is the other. Away from $L, C$ is locally the graph of a canonical transformation and the clean intersection calculus applies there.

Proposition 5.1. Assume that, away from $L, \rho$ is globally 1-1. Then

$$
\left(C \times C^{t}\right) \cap\left(T^{*} X \times \Delta_{T^{*} Y} \times T^{*} X\right)=\Delta \cup B,
$$

where $\Delta$ is the diagonal in $T^{*}(X \times Y \times Y \times X)$ and $B$ is a submanifold of dimension $2 n+k-1$. Thus, $C \circ C^{t}=\Delta \cup \Lambda_{\pi(L)}$, where $\Lambda_{\pi(L)}$ is the flow-out of $\pi(L)$.

Proof. Let $\left(m, m^{\prime}\right) \in\left(C \times C^{t}\right) \cap\left(T^{*} X \times \Delta_{T^{*} Y} \times T^{*} X\right)$ and assume that $m \notin$ $L \subset C$. Then near $m, C$ is the graph of a local canonical transformation so $\rho^{-1} \rho(m)$ and $\pi^{-1} \pi(m)$ are discrete. Since $\pi$ and $\rho$ have compact fibers, they must be finite.

Now, assume $m \in L$, then $\left(m, m^{\prime}\right) \in\left(L \times L^{t}\right) \cap\left(T^{*} X \times \Delta_{T^{*} Y} \times T^{*} X\right)$ and $m^{\prime}$ is in the integral manifold of $\Lambda_{m}=\operatorname{ker}\left(\left.d \rho\right|_{m}\right)=(d \pi)^{-1}\left\{\operatorname{Im}\left(\left.d \pi\right|_{m}\right)^{\sigma}\right\}$ passing through $m$. Thus $\left(L \times L^{t}\right) \cap\left(T^{*} X \times \Delta_{T^{*} Y} \times T^{*} X\right)$ can be written, locally, as $\bigcup_{m \in U}\{m\} \times \Lambda_{m}$, where $U$ is an open neighborhood of $m$ in $L$ and $\rho^{-1}\left(\rho\left(\phi^{-1}(m)\right)\right)$ projects down to $T^{*} X$ as the bicharacteristic of $\pi(L)$ through $m$.

Since the $\Lambda_{m}$ 's depend smoothly on $m$ and have dimension $k$, we see that $\left(L \times L^{t}\right) \cap\left(T^{*} X \times \Delta_{T^{*} Y} \times T^{*} X\right)$ is a smooth manifold of dimension $\operatorname{dim}(L)+k=$ $2 n+k-1$.

Corollary 5.2. With the notation as above $W F(A \circ B) \subset \Delta \cup \Lambda_{\pi(L)}$

As we are going to see below, much more can be said about $A \circ B$.

By means a microlocal partition of unity, we may write $A$ and $B$ as locally finite sums of operators $A=\sum_{i} A_{i}, B=\sum_{i} B_{i}$, such that each $A_{i}$ or $B_{j}$ has its wavefront set contained either in a part of $C$ where the latter is either a canonical graph or, if it intersects $L$, this occurs in a small enough neighborhood in which Theorem 4.13 applies. 
If $W F\left(A_{i}\right) \circ W F\left(B_{j}\right) \subset \Delta \backslash \Lambda_{0}$ then both $W F\left(A_{i}\right)$ and $W F\left(B_{j}\right)$ are canonical graphs and Hörmander's transverse intersection calculus applies to give $B_{j} A_{i} \in I^{m+m^{\prime}+(k-1) / 2}(\Delta) \subset I^{m+m^{\prime},-(k-1) / 2}\left(\Delta, \Lambda_{0}\right)$. The other two cases are $W F\left(A_{i}\right) \circ W F\left(B_{j}\right) \subset \Lambda_{0} \backslash \Delta$ and $W F\left(A_{i}\right) \circ W F\left(B_{j}\right) \cap \Delta \cap \Lambda_{0} \neq \varnothing$.

In the first case, we may use the clean intersection [D-G, We] calculus with excess $e=k-1$ to obtain

$$
B_{j} A_{i} \in I^{m+m^{\prime}+(k+1) / 2}\left(\Lambda_{0}\right) \subset I^{m+m^{\prime}+(k-1) / 2,-(k-1) / 2}\left(\Delta, \Lambda_{0}\right) .
$$

Finally, by Proposition 4.13, when $W F\left(A_{i}\right) \circ W F\left(B_{j}\right) \cap \Delta \cap \Lambda_{0} \neq \varnothing$; we may assume $C \subset T^{*} \mathbb{R}^{n}$ is parametrized by a phase function of the form

$$
\phi(x, y, \theta)=\sum_{i=2}^{n-k}\left(x_{i}-y_{i}\right) \theta_{i}-y_{1} \theta_{1}+\theta_{1} S\left(x, y^{\prime \prime}, \theta\right)
$$

where $(x, y, \theta) \in \mathbb{R}^{n} \times \mathbb{R}^{n} \times\left(\mathbb{R}^{n-k} \backslash 0\right)$ and $S$ satisfies (4.11) and (4.12). Let $(m, m) \in\left(C \circ C^{t}\right) \cap \Delta_{T^{*} X}$. By Hörmander's Theorem, modulo a smoothing operator, $A$ has an oscillatory representation

$$
A f(x)=\int e^{i \phi_{1}} a(x, y, \theta) f(y) d \theta d y
$$

with phase $\phi_{1}=\sum_{i=2}^{n-k}\left(x_{i}-y_{i}\right) \theta_{i}-y_{1} \theta_{1}+\theta_{1} S\left(x, y^{\prime \prime}, \theta\right)$ and an amplitude $a(x, y, \theta) \in S^{m+k / 2}\left(\mathbb{R}^{n} \times \mathbb{R}^{n} \times\left(\mathbb{R}^{n-k} \backslash 0\right)\right)$ supported in a conic neighborhood $U \times \mathbb{R}^{n-k} \backslash 0$ of $(m, m)$. Similarly $B$ has an oscillatory representation

$$
B f(z)=\int e^{-i \phi_{2}} b(z, x, \sigma) f(x) d \sigma d x
$$

with $b(z, x, \sigma) \in S^{m^{\prime}+k / 2}\left(\mathbb{R}^{n} \times \mathbb{R}^{n} \times\left(\mathbb{R}^{n-k} \backslash 0\right)\right)$ supported in $U \times \mathbb{R}^{n-k} \backslash 0$ and $\phi_{2}=\sum_{i=2}^{n-k}\left(x_{i}-z_{i}\right) \sigma_{i}-z_{1} \sigma_{1}+\sigma_{1} S\left(x, z^{\prime \prime}, \sigma\right)$. Hence the Schwartz kernel of $A B$ has the following representation:

$$
K_{A B}(z, y)=\int e^{i \psi(z, y, x, \theta, \sigma)} a \cdot b d \theta d \sigma d x,
$$

with

$$
\begin{aligned}
\psi= & \sum_{i=2}^{n-k}\left(x_{i}-y_{i}\right) \theta_{i}+z_{1} \sigma_{1}-y_{1} \theta_{1}-\sum_{i=2}^{n-k}\left(x_{i}-z_{i}\right) \sigma_{i} \\
& +\theta_{1} S\left(x, y^{\prime \prime}, \theta\right)-\sigma_{1} S\left(x, z^{\prime \prime}, \sigma\right) .
\end{aligned}
$$

We note that away from $\{|\theta|=|\sigma|\}, d_{x} \psi \neq 0$, and an integration by parts argument shows that $K_{A B}$ is a smoothing operator in this region. Thus, we may assume the Schwartz kernel of $A B$ has the form

$$
K_{A B}(z, y)=\int e^{i \psi(z, y, x, \theta, \sigma)} c(z, y, x, \theta, \sigma) d \theta d \sigma d x,
$$

where $c \in S^{m+m^{\prime}+k}\left(\mathbb{R}^{n} \times \mathbb{R}^{n} \times\left(\mathbb{R}^{n-k} \backslash 0\right)\right)$ is $a \cdot b$ supported in a conic neighborhood of $\{|\theta|=|\sigma|\}$.

Since the gradient of $\psi$ in all the variables is nowhere vanishing, integration by parts shows that expressions of the form (5.7) belong to the same fixed Sobolev space $H_{\mathrm{loc}}^{s_{0}}\left(\mathbb{R}^{n} \times \mathbb{R}^{n}\right)$ for any $s_{0}<-\left(3 n+m+m^{\prime}-k\right)$ (see, for example, [Hö3]).

The following two technical results will allow us to simplify the computations. 
Lemma 5.8. There is a neighborhood $U$ of $m$ and mappings

$$
\tilde{A}: U \rightarrow \operatorname{Hom}\left(\mathbb{R}^{n}, \mathbb{R}^{n-k}\right), \quad \tilde{B}: U \rightarrow \operatorname{Hom}\left(\mathbb{R}^{n}, \mathbb{R}^{k}\right),
$$

homogeneous of degree 0 in $(\theta, \sigma)$ such that

(1) $(\theta-\sigma) e^{i \psi}=\tilde{A} \cdot d_{x} e^{i \psi}$.

(2) $\left(z^{\prime \prime}-y^{\prime \prime}\right) \theta_{1} e^{i \psi}=\tilde{B} \cdot d_{x} e^{i \psi}$.

Proof. Near $y=z, \theta=\sigma, \theta_{1}=0$ we can write

$$
\begin{aligned}
S\left(x, y^{\prime \prime}, \theta\right)-S\left(x, z^{\prime \prime}, \sigma\right) & =\int_{0}^{1} \frac{d}{d t} S(x, t y+(1-t) z, t \theta+(1-t) \sigma) d t \\
& =\sum_{i=n-k+1}^{n}\left(y_{i}-z_{i}\right) A_{i}+\sum_{i=1}^{n-k}\left(\theta_{i}-\sigma_{i}\right) B_{i}
\end{aligned}
$$

where

$$
\begin{aligned}
& A_{i}=\int_{0}^{1} \frac{\partial S_{2}}{\partial y_{i}}(x, t y+(1-t) z, t \theta+(1-t) \sigma) d t \\
& B_{i}=\int_{0}^{1} \frac{\partial S_{2}}{\partial \theta_{i}}(x, t y+(1-t) z, t \theta+(1-t) \sigma) d t
\end{aligned}
$$

with $A_{n-k+1}, \ldots, A_{n}$ homogeneous of degree 0 and $B_{1}, \ldots, B_{n-k}$ homogeneous of degree -1 in $(\theta, \sigma)$. Hence

$$
\begin{aligned}
& \theta_{1} S\left(x, y^{\prime \prime}, \theta\right)-\sigma_{1} S\left(x, z^{\prime \prime}, \sigma\right) \\
& \quad=\theta_{1} \sum_{i=2}^{n-k}\left(\theta_{i}-\sigma_{i}\right) B_{i}+\sum_{i=n-k+1}^{n} \theta_{1}\left(y_{i}-z_{i}\right) A_{i}+\left(\theta_{1}-\sigma_{1}\right) E
\end{aligned}
$$

with $E=\theta_{1} B_{1}+S\left(x, z^{\prime \prime}, \sigma\right)$. Consider the system of equations

$$
\begin{aligned}
\frac{\partial \psi}{\partial x_{i}}- & \theta_{1} \sum_{i=2}^{n-k}\left(\theta_{i}-\sigma_{i}\right) \frac{\partial B_{i}}{\partial x_{i}} \\
= & \sum_{i=n-k+1}^{n} \theta_{1}\left(y_{i}-z_{i}\right) \frac{\partial A_{i}}{\partial x_{i}}+\left(\theta_{1}-\sigma_{1}\right) \frac{\partial E}{\partial x_{i}}, \\
& l=1, n-k+1, \ldots, n .
\end{aligned}
$$

We want to be able to solve for $\left(y_{n-k+1}-z_{n-k+1}\right) \theta_{1}, \ldots,\left(y_{n}-z_{n}\right) \theta_{1}$ and $\left(\theta_{1}-\sigma_{1}\right)$ in the system (5.11). The discriminant of this system is, by the Mean Value Theorem, the same that appears in (4.11), except that now each of the entries of the above matrix is evaluated at a point of the form $\left(x, t_{0} y+\right.$ $\left.\left(1-t_{0}\right) z, t_{0} \theta+\left(1-t_{0}\right) \sigma\right)$ for some (different for each entry) $0<t_{0}<1$. By (4.11), the determinant of this matrix is different from 0 near $y=z, \theta=\sigma$, $\theta_{1}=0$.

Thus, in a small enough neighborhood of $m$, one can solve for

$$
\left(y_{n-k+1}-z_{n-k+1}\right) \theta_{1}, \ldots,\left(y_{n}-z_{n}\right) \theta_{1}
$$


and $\left(\theta_{1}-\sigma_{1}\right)$ to obtain expressions of the form

$$
\begin{aligned}
& \theta_{1}-\sigma_{1}=C_{1} \cdot d_{x} \psi+\theta_{1} \sum_{m=2}^{n-k} D_{m}^{1}\left(\theta_{m}-\sigma_{m}\right), \\
&\left(y_{i}-z_{i}\right) \theta_{1}=C_{i} \cdot d_{x} \psi+\theta_{1} \sum_{m=2}^{n-k} D_{m}^{i}\left(\theta_{m}-\sigma_{m}\right), \\
& i=n-k+1, \ldots, n,
\end{aligned}
$$

where $C_{i} \in \operatorname{Hom}\left(\mathbb{R}^{n}, \mathbb{R}\right)$. Since

$$
\begin{aligned}
\psi= & \sum_{i=2}^{n-k}\left(x_{i}-y_{i}\right) \theta_{i}+z_{1} \sigma_{1}-y_{1} \theta_{1} \\
& -\sum_{i=2}^{n-k}\left(x_{i}-z_{i}\right) \sigma_{i}+\theta_{1} S\left(x, y^{\prime \prime}, \theta\right)-\sigma_{1} S\left(x, z_{2}, z_{3}, \sigma\right) \\
= & \sum_{i=2}^{n-k}\left(x_{i}-y_{i}\right) \theta_{i}+z_{1} \sigma_{1}-y_{1} \theta_{1}-\sum_{i=2}^{n-k}\left(x_{i}-z_{i}\right) \sigma_{i} \\
& +\theta_{1} \sum_{i=2}^{n-k}\left(\theta_{i}-\sigma_{i}\right) B_{i}+\sum_{i=n-k+1}^{n} \theta_{1}\left(y_{i}-z_{i}\right) A_{i}+\left(\theta_{1}-\sigma_{1}\right) E
\end{aligned}
$$

then, for $l=2, \ldots, n-k$,

$$
\frac{\partial \psi}{\partial x_{l}}=\theta_{l}-\sigma_{l}+\theta_{1} \sum_{i=2}^{n-k}\left(\theta_{i}-\sigma_{i}\right) \frac{\partial B_{i}}{\partial x_{l}}+\sum_{i=n-k+1}^{n} \theta_{1}\left(y_{i}-z_{i}\right) \frac{\partial A_{i}}{\partial x_{l}}+\left(\theta_{1}-\sigma_{1}\right) \frac{\partial E}{\partial x_{l}}
$$

Substituting $\left(y_{n-k+1}-z_{n-k+1}\right) \theta_{1}, \ldots,\left(y_{n}-z_{n}\right) \theta_{1}$ and the value of $\left(\theta_{1}-\sigma_{1}\right)$ in (5.12),

$$
\theta_{l}-\sigma_{l}+\theta_{1} \sum_{i=2}^{n-k} F_{i}\left(\theta_{i}-\sigma_{i}\right)+G_{l} \cdot d_{x} \psi
$$

where $G_{l} \in \operatorname{Hom}\left(\mathbb{R}^{n}, \mathbb{R}\right)$. If we regard this as a system in the unknowns $\theta_{i}-$ $\sigma_{i}, i=2, \ldots, n-k$, the determinant at $\theta_{1}=0$ is 1 . Thus in a neighborhood of $m$, one can solve for $\theta_{i}-\sigma_{i}, i=2, \ldots, n-k$, and we may write

$$
\theta_{i}-\sigma_{i}=\tilde{A}_{i} \cdot d_{x} \psi, \quad i=2, \ldots, n-k
$$

for some matrix $\tilde{A}$ with entries that are homogeneous of degree 0 in $(\theta, \sigma)$. Combining (5.12) with (5.14) proves the lemma.

Corollary 5.15. $\left(z_{1}-y_{1}\right) \theta_{1} e^{i \psi}=\left(\theta_{1} / i\right)\left(\partial / \partial \theta_{1}+\partial / \partial \sigma_{1}\right) e^{i \psi}+F \cdot d_{x} e^{i \psi}$, for some $F: \mathbb{R}^{n} \rightarrow \mathbb{R}$, homogeneous of degree 0 . 
Proof.

$$
\begin{aligned}
& \left(\frac{\partial}{\partial \theta_{1}}+\frac{\partial}{\partial \sigma_{1}}\right) \psi=z_{1}-y_{1}+\theta_{1} \sum_{i=2}^{n-k}\left(\theta_{i}-\sigma_{i}\right)\left(\frac{\partial}{\partial \theta_{1}}+\frac{\partial}{\partial \sigma_{1}}\right) B_{i} \\
& \quad+\sum_{i=n-k+1}^{n} \theta_{1}\left(y_{i}-z_{i}\right)\left(\frac{\partial}{\partial \theta_{1}}+\frac{\partial}{\partial \sigma_{1}}\right) A_{i}+\left(\theta_{1}-\sigma_{1}\right)\left(\frac{\partial}{\partial \theta_{1}}+\frac{\partial}{\partial \sigma_{1}}\right) E \\
& \quad+\sum_{i=2}^{n-k}\left(\theta_{i}-\sigma_{i}\right) B_{i}+\sum_{i=n-k+1}^{n}\left(y_{i}-z_{i}\right) A_{i}
\end{aligned}
$$

and the result follows after multiplying by $\theta_{1} e^{i \psi}$ and using Lemma 5.8 to substitute $\left(\theta_{i}-\sigma_{i}\right), i=1, \ldots, n-k$, and $\left(y_{j}-z_{j}\right) \theta_{1}, j=n-k+1, \ldots, n$.

We are now ready to make use of iterated regularity to show that if the operators $A \in I^{m}\left(\Lambda_{1}\right)$ and $B \in I^{m^{\prime}}\left(\Lambda_{1}^{t}\right)$ have Schwartz kernels $K_{A}$ and $K_{B}$ which are microlocally supported near $\Lambda_{0} \cap \Lambda_{1}$, then the Schwartz kernel of their composition $K_{A B} \in I^{p, l}\left(\Delta, \Lambda_{0}\right)$. Here, $\Delta$ denotes the diagonal in $T^{*} X \times T^{*} X$ and $\Lambda_{0}=\Lambda_{\pi(L)}$ denotes the flow-out from $\pi(L)$. Recall that near $\Delta \cap \Lambda, K_{A B}$ can be expressed as in (5.7).

By Proposition 3.4 it is enough to show that for some fixed $s_{0}$ and all firstorder pseudodifferential operators $P_{1}\left(z, D_{z} ; y, D_{y}\right), P_{2}\left(z, D_{z} ; y, D_{y}\right), \ldots$, $P_{k}\left(z, D_{z} ; y, D_{y}\right)$, whose principal symbols vanish on $\Delta^{\prime} \cup \Lambda_{0}^{\prime}$,

$$
P_{1} \cdots P_{k} u \in H_{\mathrm{loc}}^{s_{0}}(X \times X) .
$$

A set of defining functions for the diagonal, $\Delta^{\prime}$, and $\Lambda_{0}^{\prime}$ is

$$
\begin{array}{ccl}
\Delta^{\prime} & \Lambda_{\pi(L)} & \\
z_{i}-y_{i}, & z_{i}-y_{i}, & i=2, \ldots, n, \\
\zeta_{i}+\eta_{i}, & \zeta_{i}+\eta_{i}, & i=1, \ldots, n, \\
z_{i}-y_{i}, & \zeta_{i}-\eta_{i}, & i=1, n-k, \ldots, n .
\end{array}
$$

Thus a first-order pseudodifferential operator, $P\left(z, D_{z}, y, D_{y}\right)$, which is characteristic for $\Delta^{\prime} \cup \Lambda_{0}^{\prime}$ has a principal symbol which can be written in the form

$$
\begin{aligned}
p(z, y ; \zeta, \eta)= & \sum_{j=2}^{n-k}\left(z_{j}-y_{j}\right) p_{j}(z, y ; \zeta, \eta)+\sum_{j=1}^{n}\left(\zeta_{j}+\eta_{j}\right) q_{j}(z, y ; \zeta, \eta) \\
& +\sum_{i, j \in\{1, n-k+1, \ldots, n\}}\left(z_{j}-y_{j}\right)\left(\zeta_{i}-\eta_{i}\right) r_{i j}(z, y ; \zeta, \eta)
\end{aligned}
$$

where the $p_{j}$ 's, $q_{j}$ 's and $r_{i, j}$ 's are homogeneous of degrees 1,0 , and 0 , respectively. Then

$$
P K_{A B}(z, y)=\int e^{i \psi(z, y, x, \theta, \sigma)}\left(p\left(z, d_{z} \psi, y d_{y} \psi\right) c+d\right) d \theta d \sigma^{\prime} d x
$$


with $d \in S^{m+m^{\prime}+k-1}$. Note that for $l=, 2 \ldots, n-k$,

$$
\begin{aligned}
\left(\frac{\partial}{\partial \theta_{l}}+\right. & \left.\frac{\partial}{\partial \sigma_{l}}\right) \psi=z_{l}-y_{l}+\theta_{1} \sum_{i=2}^{n-k}\left(\theta_{i}-\sigma_{i}\right)\left(\frac{\partial}{\partial \theta_{l}}+\frac{\partial}{\partial \sigma_{l}}\right) B_{i} \\
& +\sum_{i=n-k+1}^{n} \theta_{1}\left(y_{i}-z_{i}\right)\left(\frac{\partial}{\partial \theta_{l}}+\frac{\partial}{\partial \sigma_{l}}\right) A_{i}+\left(\theta_{1}-\sigma_{1}\right)\left(\frac{\partial}{\partial \theta_{l}}+\frac{\partial}{\partial \sigma_{l}}\right) E
\end{aligned}
$$

where we are using the notation as in 5.13. Making use of Lemma (5.8), we can write

$$
e^{i \psi}\left(\frac{\partial}{\partial \theta_{l}}+\frac{\partial}{\partial \sigma_{l}}\right) \psi=\left(z_{l}-y_{l}\right) e^{i \psi}+e^{i \psi} H^{l}\left(d_{x} \psi\right)
$$

with $H^{l}: \mathbb{R}^{n} \rightarrow \mathbb{R}$ a matrix whose entries are homogeneous of degree -1 in $(\theta, \sigma)$.

Hence we deal with the terms $\sum_{j=2}^{n-k}\left(z_{j}-y_{j}\right) p_{j}(z, y ; \zeta, \eta)$ in $P K_{A B}(z, y)$ as follows: for $j=2, \ldots, n-k$,

$$
\begin{aligned}
& \int\left(z_{j}-y_{j}\right) e^{i \psi} p_{j}\left(z, y ; d_{z} \psi, d_{y} \psi\right) c d \theta d \sigma d x \\
& =\frac{1}{i} \int\left[\left(\frac{\partial}{\partial \theta_{j}}+\frac{\partial}{\partial \sigma_{j}}-\sum_{k=1}^{n} H_{k}^{j} \frac{\partial}{\partial x_{k}}\right) e^{i \psi}\right] p_{j}\left(z, y ; d_{z} \psi, d_{y} \psi\right) c d \theta d \sigma d x
\end{aligned}
$$

after integration by parts this becomes

$$
\begin{aligned}
= & \frac{-1}{i} \int e^{i \psi}\left[\left(\frac{\partial}{\partial \theta_{j}}+\frac{\partial}{\partial \sigma_{j}}\right) p_{j}\left(z, y ; d_{z} \psi, d_{y} \psi\right) c\right] d \theta d \sigma d x \\
& +\frac{1}{i} \sum_{k=1}^{n} \int e^{i \psi} \frac{\partial}{\partial x_{k}}\left[H_{k}^{j} p_{j}\left(z, y ; d_{z} \psi, d_{y} \psi\right) c\right] d \theta d \sigma d x
\end{aligned}
$$

But

$$
\begin{gathered}
\left(\frac{\partial}{\partial \theta_{j}}+\frac{\partial}{\partial \sigma_{j}}\right) p_{j}\left(z, y ; d_{z} \psi, d_{y} \psi\right) c \in S_{1,0}^{m+m^{\prime}+k-1} \\
\frac{\partial}{\partial x_{k}}\left[H_{k}^{j} p_{j}\left(z, y ; d_{z} \psi, d_{y} \psi\right) c\right] \in S_{1,0}^{m+m^{\prime}+k-1}
\end{gathered}
$$

for $p_{j}$ is homogeneous of degree 1 and the coefficients $H_{j}^{i}$ are homogeneous of degree -1 .

Next we study contributions of the terms $\sum_{j=1}^{n}\left(\zeta_{j}+\eta_{j}\right) q_{j}(z, y ; \zeta, \eta)$. Making use of Lemma 5.8, we note, first, that, for $j=1, \ldots, n-k$, the leading coefficient coming from the term $\left(\zeta_{j}+\eta_{j}\right) q_{j}(z, y ; \zeta, \eta)$ is

$$
\begin{gathered}
\int\left(\left(\frac{\partial}{\partial z_{j}}+\frac{\partial}{\partial y_{j}}\right) \psi\right) e^{i \psi} q_{j}\left(z, y ; d_{z} \psi, d_{y} \psi\right) c d \theta d \theta d \sigma d \sigma d x \\
\quad=\int\left(\sigma_{j}-\theta_{j}\right) e^{i \psi} q_{j}\left(z, y ; d_{z} \psi, d_{y} \psi\right) c d \theta_{j} d \theta d \sigma d \sigma d x \\
\quad=\frac{j}{i} \int \tilde{B}_{j} d_{x}\left(e^{i \psi}\right) q_{j}\left(z, y ; d_{z} \psi, d_{y} \psi\right) c d \theta d \theta d \sigma d \sigma d x
\end{gathered}
$$


whereas for $j=n-k+1, \ldots, n$ the leading coefficient coming from the term $\left(\zeta_{j}-\eta_{j}\right) q_{j}(z, y ; \zeta, \eta)$ is

$$
\begin{aligned}
& \int\left[\left(\frac{\partial}{\partial z_{j}}+\frac{\partial}{\partial y_{j}}\right) \psi\right] e^{i \psi} q_{j} c d \theta d \sigma d x=\int\left(\theta_{1} \sum_{i=2}^{n-k}\left(\theta_{i}-\sigma_{i}\right)\left(\frac{\partial}{\partial z_{j}}+\frac{\partial}{\partial y_{j}}\right) B_{i}\right. \\
& \quad+\sum_{i=n-k+1}^{n} \theta_{1}\left(y_{i}-z_{i}\right)\left(\frac{\partial}{\partial z_{j}}+\frac{\partial}{\partial y_{j}}\right) A_{i} \\
& \quad+\left(\theta_{1}-\sigma_{1}\right)\left(\frac{\partial}{\partial z_{j}}+\frac{\partial}{\partial y_{j}}\right) E e^{i \psi} q_{j} c d \theta d \sigma d x,
\end{aligned}
$$

and we may substitute $\left(\theta_{i}-\sigma_{i}\right), i=1, \ldots, n$, and $\theta_{1}\left(y_{i}-z_{i}\right), i=n-k+$ $1, \ldots, n$, by the value given in Lemma 5.8 leading to an expression of the form

$$
\int G \cdot d_{x}\left(e^{i \psi}\right) q_{j}\left(z, y ; d_{z} \psi, d_{y} \psi\right) c d \theta d \sigma d x
$$

for some matrix valued function $G$ whose entries are homogeneous of degree 0 . After integration by parts we see that both terms are again of the form (5.7).

We now look at the contributions of the terms $\left(z_{j}-y_{j}\right)\left(\zeta_{i}-\eta_{i}\right) r_{i j}(z, y ; \zeta, \eta)$, for $i, j \in\{1, n-k+1, \ldots, n\}$.

The leading term in $P K_{A B}$ coming from $\left(z_{1}-y_{1}\right)\left(\zeta_{1}-\eta_{1}\right) r_{11}(z, y ; \zeta, \eta)$ is

$$
\begin{gathered}
\int\left(z_{1}-y_{1}\right)\left[\left(\frac{\partial}{\partial z_{1}}-\frac{\partial}{\partial y_{1}}\right) \psi\right] e^{i \psi} r_{11}\left(z, y ; d_{z} \psi, d_{y} \psi\right) c d \theta_{1} d \theta d \sigma_{1} d \sigma d x \\
=\int\left(z_{1}-y_{1}\right)\left(\sigma_{1}+\theta_{1}\right) e^{i \psi} r_{11} c d \theta d \sigma d x
\end{gathered}
$$

but from Lemma $5.8 \sigma_{1} e^{i \psi}=\theta_{1}-\tilde{B}_{1} \cdot d_{x} e^{i \psi}$, so this is equal to

$$
2 \int\left(z_{1}-y_{1}\right) \theta_{1} e^{i \psi} r_{11} c d \theta_{1} d \theta d \sigma_{1} d \sigma d x-\int\left(z_{1}-y_{1}\right) \tilde{B} \cdot d_{x} e^{i \psi} r_{11} c d \theta d \sigma d x
$$

Now, by Corollary 5.15 above, this is

$$
\begin{aligned}
2 \int & \left(\frac{\theta_{1}}{i}\left(\frac{\partial}{\partial \theta_{1}}+\frac{\partial}{\partial \sigma_{1}}\right) e^{i \psi}+F \cdot d_{x} e^{i \psi} r_{11} c\right) d \theta_{1} d \theta d \sigma_{1} d \sigma d x \\
& -\int\left(z_{1}-y_{1}\right) \tilde{B} \cdot d_{x} e^{i \psi} r_{11} c d \theta d \sigma d x
\end{aligned}
$$

which after integration by parts has again the same homogeneity as in (5.7).

The terms $\left(z_{i}-y_{i}\right)\left(\zeta_{1}-\eta_{1}\right) r_{1 i}(z, y ; \zeta, \eta), j \geq n-k+1$, can be treated in a similar way, except that the computations are easier since one can apply directly Lemma 5.8 .

Finally, the main contribution to the integrand coming from terms of the 
form $\left(z_{i}-y_{i}\right)\left(\zeta_{j}-\eta_{j}\right) r_{i j}(z, y ; \zeta, \eta), \quad j \geq n-k+1$, is

$$
\begin{aligned}
\left(z_{i}-y_{i}\right)\left[\left(\frac{\partial}{\partial z_{j}}-\frac{\partial}{\partial y_{j}}\right) \psi\right] & \left(z_{i}-y_{i}\right)\left(\theta_{1} \sum_{i=2}^{n-k}\left(\theta_{i}-\sigma_{i}\right)\left(\frac{\partial}{\partial z_{j}}-\frac{\partial}{\partial y_{j}}\right) B_{i}\right. \\
& +\sum_{i=n-k+1}^{n} \theta_{1}\left(y_{i}-z_{i}\right)\left(\frac{\partial}{\partial z_{j}}-\frac{\partial}{\partial y_{j}}\right) A_{i} \\
& \left.+\left(\theta_{1}-\sigma_{1}\right)\left(\frac{\partial}{\partial z_{j}}-\frac{\partial}{\partial y_{j}}\right) E\right),
\end{aligned}
$$

and, again, we can use Lemma 5.8 to substitute $\left(\theta_{i}-\sigma_{i}\right), i=1, \ldots, n-k$, $\theta_{1}\left(y_{j}-z_{j}\right), j=n-k+1, \ldots, n$, allowing one to reduce the integrand to the appropriate homogeneity after integration by parts.

From these computations the next theorem follows easily.

Theorem 5.18. Let $C \subset T^{*}(X \backslash 0) \times T^{*}(Y \backslash 0)$ be as in Theorem 4.13 and let $A \in I^{m}(C), B \in I^{m^{\prime}}\left(C^{t}\right)$ be properly supported Fourier integral operators. Then $B A \in I^{m+m^{\prime}+(k-1) / 2,-(k-1) / 2}\left(\Delta_{T^{*} X}, \Lambda_{\pi(L)}\right)$.

Proof. The method of iterated regularity above and Proposition 3.4 show that $B A \in I^{p, l}\left(\Delta, \Lambda_{2}\right)$ for some $p$, and $l$. To determine these orders, we note that away from $L=\left\{\theta_{1}=0\right\}$ the composition is covered by Hörmander's calculus, and hence, $B A \in I^{m+m^{\prime}}\left(\Delta_{T^{*} X} \backslash \Lambda_{\pi(L)}\right) \subset I^{m+m^{\prime}, 0}\left(\Delta_{T^{*} X}, \Lambda_{\pi(L)}\right)$. Thus $p+l=m+m^{\prime}$.

If $a$ is the principal symbol of $A$, considered as a $\frac{1}{2}$-density on $\Delta_{T^{*} X}$, one can write $a$ as $\alpha \times\left|\pi^{*} \omega_{X}^{n}\right|^{1 / 2}$, where $\omega_{X}$ is the canonical symplectic form in $T^{*} X$ and $\pi$ denotes the projection from $C$ onto $T^{*} X$. Since $\pi$ drops rank by $k$ at $L, \pi^{*} \omega^{n}$ vanishes to $k$ th order at $L$ an $\alpha$ has a singularity at $L$ of order $-k / 2$.

Similarly if $b$ denotes the principal symbol of $B$, then $b=\beta \times\left|\pi^{*} \omega_{X}^{n}\right|^{1 / 2}$ with $\beta$ having at $L$ a singularity of order $-k / 2$.

Thus $\left.\beta \cdot \alpha\right|_{T^{*} X \times \Delta_{T^{*} X} \times T^{*} X}$ has a singularity, above $\pi(L)$, of order $-k$. When pushed down by $\pi$, it still gives rise to a singularity of the same order at $L$, in the principal symbol $\beta \times \alpha$ of $B A$. Hence, by [Gu-U, Proposition 5.4], $-k=l-(k+1) / 2$, so $l=(1-k) / 2$ and $p=m+m^{\prime}+(k-1) / 2$.

We recall the following estimate from [G-U2],

Proposition 5.19. Let $\Sigma \subset T^{*} M \backslash 0$ be a smooth, conic, codimension $j$ submanifold and $\Lambda \subset\left(T^{*} M \backslash 0\right) \times\left(T^{*} M \backslash 0\right)$ its flow-out. Then, if $\max (p+j / 2, p+l) \leq$ $-s_{0}$ and $T \in I^{p, l}(\Delta, \Lambda)$, for each $s \in \mathbb{R}$, we have that $T: H_{\text {comp }}^{s}(M) \rightarrow$ $H_{\text {loc }}^{s+s_{0}}(M)$.

Combining the two previous results we obtain

Corollary 5.20. For $A$ as above, $A: H^{s}(X) \rightarrow H_{\mathrm{loc}}^{s-m-k / 2}(X)$ is bounded.

Proof. From the theorem we have that $A^{*} A \in I^{2 m+(k-1) / 2,(1-k) / 2}\left(\Delta_{T^{*} X}, \Lambda_{\pi(L)}\right)$ is properly supported, so from 5.19 it maps $A A^{*}: H^{s}(X) \rightarrow H_{\mathrm{loc}}^{s-2 m-k}(X)$. 
Hence, the corollary follows for $s=m+k / 2$. For general $s \in \mathbb{R}$ apply this result to $P A Q$, where $P$ and $Q$ are elliptic pseudodifferential operators on $X$ and $Y$ of orders $s-m-(k+1) / 2$ and $-s+m+(k+1) / 2$, respectively.

Note that the above estimate is no better than the ones that follow from [Hö3], even though we are in a less singular case. Example 2.15 shows that the loss of $k / 2$ derivatives is sharp. There, the Lagrangian with both projections blow-downs, dropping rank by $k=2$, is the conormal bundle of a codimension 4 submanifold, say $M$. The FIO associated with surface measure on $M$ is of order -1 and it is easy to see that it is no better than bounded in $L^{2}$.

We have the following compositions: $\Lambda_{\pi(L)}^{t} \circ \Lambda_{\pi(L)}=C^{t} \circ \Lambda_{\pi(L)}=\Lambda_{\pi(L)}^{t} \circ C=$ $C, C^{t} \circ C=\Delta \cup \Lambda_{\pi(L)}$. Thus $\left(C \cup \Lambda_{\pi(L)}\right)^{t} \circ\left(C \cup \Lambda_{\pi(L)}\right)=\Delta \cup \Lambda_{\pi(L)}$ does not yield new Lagrangians. It is then natural to ask whether the above theorem also holds in the $I^{p, l}$ class. The method above can be extended to prove

Theorem 5.21. Let $C \subset T^{*}(X \backslash 0) \times T^{*}(Y \backslash 0)$ and $\Lambda_{\pi(L)}$ be as above. Let $A \in I^{p, l}\left(C, \Lambda_{\pi(L)}\right), B \in I^{r, s}\left(C^{t}, \Lambda_{\pi(L)}^{t}\right)$ be properly supported. Then $B \circ A \in$ $I^{p+r+(k-1) / 2,-(k-1) / 2}\left(\Delta_{T^{*} X}, \Lambda_{\pi(L)}\right)$.

Proof. The proof is a slight variation of Theorem 5.18. We will only give here the modifications and leave the details to the reader. Let $A \in I^{p, l}\left(C, \Lambda_{\pi(L)}\right)$, $B \in I^{r, s}\left(C^{t}, \Lambda_{\pi(L)}^{t}\right)$. As in the previous theorem, after a microlocal partition of unity, it is enough to consider a composition $B \circ A$, where $C, \Lambda_{\pi(L)} \subset$ $T^{*}\left(\mathbb{R}^{n} \times \mathbb{R}^{n}\right)$ and $A$ and $B$ are supported near a point $m \in C \cap \Lambda_{\pi(L)}$.

From 4.13 and 3.6, distributions in $I^{p, l}\left(C, \Lambda_{\pi(L)}\right)$ can be represented using a multiphase

$$
\Phi(x, y, t, \omega)=\sum_{i=2}^{n-k}\left(x_{i}-y_{i}\right) \omega_{i}-t|\omega|\left(y_{1}+S\left(x, y^{\prime \prime}, t|\omega|, \omega\right)\right),
$$

where $S(x, y, \theta)$ satisfies (4.11) and (4.12) with $\theta=(t, \omega)$. Hence we may write the Schwartz kernel, $u(x, y)$, of $A$ as

$$
u(x, y)=\iint_{{\mathbf{R} \times \mathbf{R}^{n-k-1}}} e^{i \phi_{1}} a(x, y, t, \omega) d t d \omega,
$$

with a phase $\phi_{1}=\sum_{i=2}^{n-k}\left(x_{i}-y_{i}\right) \omega_{i}-t\left(y_{1}+S\left(x, y^{\prime \prime}, t, \omega\right)\right)$ and a product type amplitude, $a(x, y, t, \omega)$, of order $p+l-(n-1) / 2$ in $\omega$ and $-l+(n+k-1) / 2$ in $t$. For convenience, we are writing $\omega=\left(\omega_{2}, \ldots, \omega_{n-k}\right) \in \mathbb{R}^{n-k-1}$. Note that the only contributions to the wavefront set of $u$ near $C \cap \Lambda_{\pi(L)}$, come from a conic neighborhood of $\{t=0\}$. Thus, we may assume integration takes place in the region $\{|t|<|\omega|\}$.

Similarly, the Schwartz kernel of $B$ has an expression of the form

$$
v(x, y)=\iint_{\mathbf{R} \times \mathbb{R}^{n-k-1}} e^{-i \phi_{2}} b(z, x, \delta, \sigma) d \delta d \sigma,
$$

where $\phi_{2}=\sum_{i=2}^{n-k}\left(x_{i}-z_{i}\right) \sigma_{i}-\delta\left(z_{1}+S\left(x, z^{\prime \prime}, \delta, \sigma\right)\right)$ and $b(z, x, \delta, \sigma)$ is a product type symbol of order $r+s-(n-1) / 2$ in $\sigma$ and $-s+(n+k-1) / 2$ in $\delta$ and the integral is extended to the region $\{|\delta|<|\sigma|\}$. 
As in the argument preceding Theorem 5.18 we may assume that, modulo lower order contributions, the Schwartz kernel of $B \circ A$ has an oscillatory integral representation of the form

$$
K_{A B}(z, y)=\int e^{i \psi(z, y, x, \omega, \sigma, t, \delta)} c(x, y, \omega, \sigma, t, \delta) d \omega d \sigma d t d \delta d x
$$

where integration takes place in a conic neighborhood of the region $\{|\omega|=$ $|\sigma|, \quad t=|\delta|\} \cap\{|t|<|\omega|\} \cap\{|\delta|<|\sigma|\}$, the phase is

$$
\begin{aligned}
\psi= & \sum_{i=2}^{n}\left(x_{i}-y_{i}\right) \omega_{i}-\sum_{i=2}^{n}\left(x_{i}-z_{i}\right) \sigma_{i} \\
& +\delta\left(S\left(x, y^{\prime \prime}, \omega\right)-y_{1}\right)-t\left(S\left(x, z^{\prime \prime}, \sigma^{\prime}\right)-z_{1}\right) .
\end{aligned}
$$

and $c(x, y, \omega, \sigma, t, \delta)$ is a product type symbol of order $p+l+r+s-n+1$ in $(\omega, \sigma)$ and $n+k-l-s-1$ in $(t, \delta)$.

But, in the region of integration, $c(x, y, \omega, \sigma, t, \delta)$ behaves like a regular symbol of type $p+r+k$ in the combined variables $(\sigma, \omega, t, \delta)$.

In this situation, the method of iterated regularity may be used as in 5.18 to yield $A \circ B \in I^{p^{\prime}}, l^{\prime}$ for some $p^{\prime}, l^{\prime}$. To compute the values of $p^{\prime}$ and $l^{\prime}$, we observe that the only contribution to $\Delta$ comes from $C^{t} \circ C$. Away from $\Lambda_{\pi(L)}$ we have, according to [Gu-U], that $A \in I^{p}\left(C \backslash \Lambda_{\pi(L)}\right)$ and $B \in I^{r}\left(C^{t} \backslash \Lambda_{\pi(L)}\right)$.

On $C \backslash \Lambda_{\pi(L)}$ Hörmander's transverse composition calculus applies. Then $\left.B \circ A\right|_{\Delta \backslash \Lambda_{x(L)}} \in I^{p+r}\left(\Delta \backslash \Lambda_{\pi(L)}\right)$ and, as in 5.18, $\left.\sigma(B \circ A)\right|_{\Delta \backslash \Lambda_{\mathbf{x}(L)}}$ has a conormal singularity of order $-k$ at $\pi(L)$. Hence, we have that $p^{\prime}+l^{\prime}=p+r$ and $l^{\prime}=-(k-1) / 2$.

The same proof as in Corollary 5.20 now yields

Corollary 5.24. For $A$ as in (5.21), $A: H^{s}(X) \rightarrow H_{\mathrm{loc}}^{s-(p+l+k) / 2}(X)$ is bounded.

\section{REFERENCES}

[A-U] J. L. Antoniano and G. A. Uhlmann, A functional calculus for a class of pseudodifferential operators with singular symbols, Proc. Sympos. Pure Math., vol. 43, Amer. Math. Soc., Providence, RI, 1985, pp. 5-16, 5-16.

[Be] M. Berger, P. Godouchon and E. Mazet, Le spectre d'une variété Riemannienne, Lecture Notes in Math., vol. 194, Springer-Verlag, Berlin and New York, 1971.

[Bo] R. Bott, On the iteration of closed geodesics and the Sturm intersection theory, Comm. Pure Appl. Math. 9 (1956), 171-206.

[DeT-G1] D. M. DeTurck and C. S. Gordon, Isospectral deformations. I: Riemannian structures on two-step nilspaces, Comm. Pure Appl. Math. 40 (1987), 367-387.

[DeT-G2] __, Isospectral deformations. II: Trace formulas, metrics and potentials, Comm. Pure Appl. Math. 42 (1989), 1067-1095.

[D] J. J. Duistermaat, Fourier integral operators, Courant Institute, New York, 1973.

[D-G] J. J. Duistermaat and V. Guillemin, The spectrum of positive elliptic operators and periodic bicharacteristics, Invent. Math. 29 (1975), 39-79.

[G-W] C. S. Gordon and E. Wilson, Isospectral deformations of compact solvmanifolds, J. Differential Geom. 19 (1984), 241-256.

[G-U1] A. Greenleaf and G. Uhlmann, Nonlocal inversion formulas for the X-ray transform, Duke Math. J. 58 (1989), 205-240.

[G-U2] _ Estimates for singular Radon transforms and pseudodifferential operators with singular symbols, J. Funct. Anal. 89 (1990), 202-232. 
[G-U3] Composition of some singular Fourier integral operators and estimates for restricted $X$-ray transforms, Ann. Inst. Fourier (Grenoble) 40 (1990), 443-466.

[G-U4] , private communication.

[Gu1] V. Guillemin, Cosmology in (2+1)-dimensions, cyclic models and deformations, Princeton Univ. Press, Princeton, NJ, 1989.

[Gu-U] V. Guillemin and G. Uhlmann, Oscillatory integrals with singular symbols, Duke Math. J. 48 (1981), 251-267.

[Hö1] L. Hörmander, The analysis of linear partial differential operators III, Springer-Verlag, Berlin and New York, Germany, 1985.

[Hö2] _ The analysis of linear partial differential operators IV, Springer-Verlag, Berlin and New York, Germany, 1985.

[Hö3] — Fourier integral operators I, Acta Math. 127 (1971), 79-183.

[Ma] F. Marhuenda, Microlocal analysis of some isospectral problems, Ph.D. thesis, Univ. Rochester, New York, 1990.

[Me1] R. Melrose, Transformation of boundary problems, Acta Math. 147 (1981), 149-236.

[Me2] _ Marked Lagrangians, Notes of lectures at Max Planck Institut, 1987, paper in preparation.

[M-U] R. Melrose and G. Uhlmann, Lagrangian intersection and the Cauchy problem, Comm. Pure Appl. Math. 32 (1979), 482-519.

[Mn] G. Mendoza, Symbol calculus associated with intersecting Lagrangians, Comm. Partial Differential Equations 7 (1982), 1035-1116.

[T] F. Treves, Introduction to pseudodifferential and Fourier integral operators, Plenum, New York, 1980.

[We] A. Weinstein, On Maslov's quantization condition, Fourier Integral Operators and Partial Differential Equations, (J. Chazarain, ed.), Springer-Verlag, Berlin and New York, 1975, pp. 341-372.

[Z] S. Zelditch, Isospectrality in the category of Fourier integral operators. I, preprint.

Departamento Fundamentos Analisis Economico, University of Alicante, 03071 AliCANTE, SPAIN

E-mail address: jaume@vm.cpd.ua.es; jaume@ealiun11 